\title{
Dually hemimorphic semi-Nelson algebras
}

\author{
JUAN MANUEL CORNEJO*, Departamento de Matemática, Universidad \\ Nacional del Sur and Instituto de Matemática de Bahía Blanca (INMABB) - \\ Consejo Nacional de Investigaciones Científicas y Técnicas, Av. Leandro N. Alem \\ $N^{o} 1253$ - $2^{\circ}$ Piso, Bahía Blanca 8000, Argentina.
}

\author{
HERNÁN JAVIER SAN MARTÍN**, Departamento de Matemática, Facultad de \\ Ciencias Exactas and Consejo Nacional de Investigaciones Cientificas y Técnicas, \\ Casilla de correos 172, La Plata 1900, Argentina.
}

\begin{abstract}
Extending the relation between semi-Heyting algebras and semi-Nelson algebras to dually hemimorphic semi-Heyting algebras, we introduce and study the variety of dually hemimorphic semi-Nelson algebras and some of its subvarieties. In particular, we prove that the category of dually hemimorphic semi-Heyting algebras is equivalent to the category of dually hemimorphic centered semi-Nelson algebras. We also study the lattice of congruences of a dually hemimorphic semi-Nelson algebra through some of its deductive systems.
\end{abstract}

Keywords: Semi-Heyting algebras, semi-Nelson algebras, expansions, categorical equivalence.

\section{Introduction}

In 1942 'modal symmetric propositional calculus' was introduced by Moisil [13]. It is the extension of the positive calculus of Hilbert-Bernays obtained by adding as new connective the De Morgan's negation ', which verifies the two axioms $\alpha \rightarrow \alpha^{\prime \prime}, \alpha^{\prime \prime} \rightarrow \alpha$ and the following contraposition rule: if $\alpha \rightarrow \beta$ then $\beta^{\prime} \rightarrow \alpha^{\prime}$. In 1980 Monteiro introduced the structure of 'symmetric Heyting algebras' [14]. An algebra $\left(A, \wedge, \vee, \rightarrow,,^{\prime}, 0,1\right)$ is said to be a symmetric Heyting algebra if $(A, \wedge, \vee, \rightarrow, 0,1)$ is a Heyting algebra and $\left(A, \wedge, \vee,{ }^{\prime}, 0,1\right)$ is a De Morgan algebra. In the mentioned paper Monteiro proved an algebraic completeness theorem for the modal symmetric propositional calculus by showing that the variety of symmetric Heyting algebras is its equivalent semantics. In 1987 this variety was also studied by Sankappanavar [20], among others.

Later, in 2008, Sankappanavar introduced and studied semi-Heyting algebras as an abstraction of Heyting algebras [21]. There already exists some literature related to this variety. The papers that deal with this variety from an algebraic point of view include $[1-4,7,21]$ and the papers that deal with logical approaches include [5, 6, 9]. Then, in 2011, Sankappanavar introduced a variety of algebras called 'dually hemimorphic semi-Heyting algebras' as expansions of semi-Heyting algebras by a dual hemimorphism, which is a common generalization of De Morgan operation and the dual pseudocomplementation [19]. This variety was studied logically in a recent work [8].

Nelson algebras were defined by Rasiowa [17]. The class of Nelson algebras, which is a variety, is the algebraic semantics of the intuitionistic propositional calculus with strong negation introduced by Nelson [15]. There is a close relationship between Nelson algebras and Heyting algebras, as it

\footnotetext{
*E-mail: jmcornejo@uns.edu.ar

**E-mail: hsanmartin@mate.unlp.edu.ar
}

Vol. 00, No. 0, (C) The Author(s) 2019. Published by Oxford University Press. All rights reserved. For permissions, please e-mail: journals.permission@oup.com. 


\section{Dually Hemimorphic Semi-Nelson Algebras}

was investigated by Vakarelov [24] and Sendlewski [22], among others. This relationship is part of what is now known as twist structures [12, 16, 18] and date back to [11]. In [10] Cornejo and Viglizzo extended the twist construction to semi-Heyting algebras obtaining a new variety, which they called semi-Nelson algebras. They considered semi-Heyting algebras and introduced semi-Nelson algebras, showing that some of the features of the original constructions between Heyting algebras and Nelson algebras given by Vakarelov are preserved [24]. More precisely, the following properties were proved in [10]: (i) if $\mathbf{A}$ is a semi-Heyting algebra then we can define a semi-Nelson algebra, $\mathbf{F}(\mathbf{A})$, and if $\mathbf{B}$ is a semi-Nelson algebra then we can define a semi-Heyting algebra, $\mathbf{G}(\mathbf{B})$; (ii) if $\mathbf{A}$ is a semi-Heyting algebra then there exists an isomorphism between $\mathbf{A}$ and $G(F(A))$, and if $\mathbf{B}$ is a semi-Nelson algebra then $\mathbf{B}$ is isomorphic to a subalgebra of $F(G(B))$. In [10] the authors also characterized the lattice of congruences of a semi-Nelson algebra and they used it in order to prove that the variety of semi-Nelson algebras is arithmetical, has equationally definable principal congruences and has the congruence extension property.

The main goal of this paper is to extend the relation between semi-Heyting algebras and semiNelson algebras [10] in the framework of dually hemimorphic semi-Heyting algebras and some of its subvarieties [19]. The paper is structured as follows. In Section 2 we recall the definition of the variety of dually hemimorphic semi-Heyting algebras and some of its subvarieties, as well the relation between them. In Section 3 we introduce and study the variety of dually hemimorphic semi-Nelson algebras and some of its subvarieties. In Section 4 we explore the relation between dually hemimorphic semi-Heyting algebras and dually hemimorphic semi-Nelson algebras. In particular, we prove that the category of dually hemimorphic semi-Heyting algebras is equivalent to the category of dually hemimorphic centered semi-Nelson algebras. In Section 5 we study the lattice of congruences of any dually hemimorphic semi-Nelson algebra. Finally, in Section 6 we present an alternative construction of the equivalence for the category of dually hemimorphic centered semi-Heyting algebras.

\section{Preliminaries}

Throughout this paper we will use the same notation for a class of algebras and its corresponding (algebraic) category.

\section{DEFINITION 2.1}

An algebra $(A, \wedge, \vee, \rightarrow, 0,1)$ of type $(2,2,2,0,0)$ is a semi-Heyting algebra if $(A, \wedge, \vee, 0,1)$ is a bounded lattice and the following equations are satisfied:

$(E 1): \quad x \wedge(x \rightarrow y)=x \wedge y$,

$(E 2): \quad x \wedge(y \rightarrow z)=x \wedge[(x \wedge y) \rightarrow(x \wedge z)]$,

(E3): $x \rightarrow x=1$.

We write $\mathbb{S H}$ for the variety of semi-Heyting algebras. The underlying lattice of a semiHeyting algebra is necessarily distributive, as it is shown in [21]. Semi-Heyting algebras share with Heyting algebras the following properties: they are pseudocomplemented and their congruences are determined by the lattice filters. The relationship between the variety of semi-Heyting algebras and the varieties of Heyting algebras (and its expansions) has been also studied in [1-4, 19]. In [21] it was proved that Heyting algebras are semi-Heyting algebras, which satisfy the equation $(x \wedge y) \rightarrow x=1$.

Now we give the definition of dually hemimorphic semi-Heyting algebra, which was introduced by Sankappanavar in [19].

DEFINITION 2.2

An algebra $\left(A, \wedge, \vee, \rightarrow,^{\prime}, 0,1\right)$ of type $(2,2,2,1,0,0)$ is said to be a dually hemimorphic 
semi-Heyting algebra if $(A, \wedge, \vee, \rightarrow, 0,1)$ is a semi-Heyting algebra and the following equations are satisfied:

$(E 4): \quad 0^{\prime}=1$,

$(E 5): 1^{\prime}=0$,

(E6): $\quad(x \wedge y)^{\prime}=x^{\prime} \vee y^{\prime}$

We write $\mathbb{D H M} \mathbb{M} H$ to indicate the variety of dually hemimorphic semi-Heyting algebras.

Let $\left(A, \wedge, \vee, \rightarrow,^{\prime}, 0,1\right)$ be an algebra of type $(2,2,2,1,0,0)$. To improve the readability of this paper we expose the following list of equations:

(E7): $\quad(x \wedge y) \rightarrow x=1$

$(E 8): \quad(x \vee y)^{\prime}=x^{\prime} \wedge y^{\prime}$

$(E 9): x^{\prime \prime} \leq x$

(E10): $\quad x^{\prime \prime}=x$

$(E 11): \quad(x \vee y)^{\prime \prime}=x^{\prime \prime} \vee y^{\prime \prime}$

$(E 12): \quad x^{\prime \prime \prime}=x^{\prime}$

(E13): $\quad x \vee x^{\prime}=1$

$(E 14): \quad(x \vee(x \rightarrow 0))^{\prime}=x^{\prime} \wedge(x \rightarrow 0)^{\prime}$

$(E 15): \quad x \vee(x \rightarrow 0)=1$

(E16): $\left(x \vee y^{\prime}\right)^{\prime}=x^{\prime} \wedge y^{\prime \prime}$

(E17): $\quad x^{\prime} \wedge x^{\prime \prime}=0$.

We also list the following sets formed by the above equations:

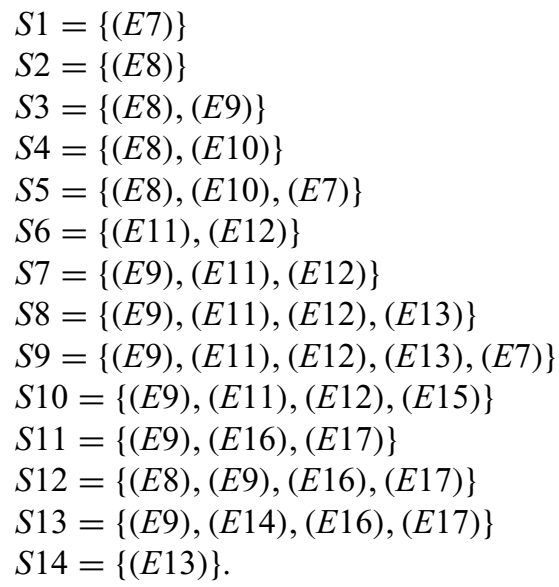

To avoid confusions we will present the definitions of some subvarieties of $\mathbb{D H M} \mathbb{M}$ by using a table. These classes of algebras were introduced in [19].

\section{DEFINITION 2.3}

The Figure 1 represents how several subvarieties of $\mathbb{D H M} \mathbb{M} \mathbb{H}$ are defined in [19] where the first column indicates the name of the class, the second column indicates the defining set of equations modulo $\mathbb{D H M} \mathbb{M} \mathbb{H}$ and the third column indicates the notation along this paper.

Most of the proofs of the items in the below lemma could be found in [19]. The rest are straightforward. 


\section{Dually Hemimorphic Semi-Nelson Algebras}

\begin{tabular}{l|c|c} 
Class of algebras & Def. set & Notation \\
\hline Dually hemimorphic Heyting algebras & $S 1$ & $\mathbb{D H M H}$ \\
Semi Heyting algebras with an Ockham operation & $S 2$ & $\mathbb{O C K S H}$ \\
Semi Heyting algebras with a dual MS-operation & $S 3$ & $\mathbb{D} m s \mathbb{S H}$ \\
De Morgan (or symmetric) semi-Heyting algebras & $S 4$ & $\mathbb{D M S H}$ \\
De Morgan (or symmetric) Heyting algebras & $S 5$ & $\mathbb{D M} \mathbb{H}$ \\
Semi-Heyting algebras with a dual semi-De Morgan operation & $S 6$ & $\mathbb{D S D S H}$ \\
Dually quasi-De Morgan semi-Heyting algebras & $S 7$ & $\mathbb{D Q D S H}$ \\
Semi Heyting algebras with a dual pseudocomplementation & $S 8$ & $\mathbb{D P C S H}$ \\
Heyting algebras with a dual pseudocomplementation & $S 9$ & $\mathbb{P} \mathbb{C H}$ \\
Boolean semi Heyting algebras with a dual quasi De Morgan operation & $S 10$ & $\mathbb{D Q D B S H}$ \\
Semi Heyting algebras with a dual quasi Stone operation & $S 11$ & $\mathbb{D Q S S H}$ \\
Semi Heyting algebras with a dual Stone operation & $S 12$ & $\mathbb{D S S H}$ \\
Semi Heyting algebras with a blended dual quasi Stone operation & $S 13$ & $\mathbb{B D Q S S H I}$ \\
Semi Heyting algebras with a dual semicomplementation & $S 14$ & $\mathbb{D S C S H I}$
\end{tabular}

FIGURE 1. Subvarieties of $\mathbb{D H M} \mathbb{M}$ H.

LEMMA 2.4

The following inclusions are proper:

(1) $\mathbb{D M H} \subseteq \mathbb{D M S H} \subseteq \mathbb{D} m s \mathbb{S H} \subseteq \mathbb{O C K S H} \subseteq \mathbb{D H M S H}$,

(2) $\mathbb{D P C H} \subseteq \mathbb{D P C S H} \subseteq \mathbb{D Q D S H} \subseteq \mathbb{D S D S H} \subseteq \mathbb{D H M} \mathbb{M} H$,

(3) $\mathbb{D Q D B S H} \subseteq \mathbb{D Q D S H}$,

(4) $\mathbb{D S S H} \subseteq \mathbb{D Q S S H}$,

(5) $\mathbb{B D Q Q S S H} \subseteq \mathbb{D Q S S H}$,

(6) $\mathbb{D P C H} \subseteq \mathbb{D H M} \mathbb{M} \subseteq \mathbb{D H M} \mathbb{H}$,

(7) $\mathbb{D M M} \subseteq \mathbb{D} \mathbb{H M} \mathbb{H}$,

(8) $\quad \mathbb{D S C S H} \subseteq \mathbb{D H M} \mathbb{H} H$.

\section{Dually hemimorphic semi-Nelson algebras}

In this section we introduce the variety of dually hemimorphic semi-Nelson algebras and some of its subvarieties. The motivation to introduce this variety will be justified in the next section, when we study its connection with the variety of dually hemimorphic semi-Heyting algebras.

Let $\left(A, \wedge, \vee, \rightarrow,^{\prime}, 0,1\right)$ be an algebra of type $(2,2,2,1,0,0)$. We define the binary map $\rightarrow_{N}$ on $A$ by

$$
x \rightarrow{ }_{N} y:=x \rightarrow(x \wedge y) .
$$

In order to improve the readability of this section we will expose the following list of equations:

$(E 18): \quad x \wedge(x \vee y)=x$,

(E19): $\quad x \wedge(y \vee z)=(z \wedge x) \vee(y \wedge x)$

$(E 20): \sim \sim x=x$

$(E 21): \sim(x \wedge y)=\sim x \vee \sim y$,

$(E 22): \quad x \wedge \sim x=(x \wedge \sim x) \wedge(y \vee \sim y)$,

$(E 23): x \wedge\left(x \rightarrow_{N} y\right)=x \wedge(\sim x \vee y)$,

$(E 24): x \rightarrow_{N}\left(y \rightarrow_{N} z\right)=(x \wedge y) \rightarrow_{N} z$

$(E 25): \quad\left(x \rightarrow_{N} y\right) \rightarrow_{N}\left[\left(y \rightarrow_{N} x\right) \rightarrow_{N}\left[(x \rightarrow z) \rightarrow_{N}(y \rightarrow z)\right]\right]=1$,

$(E 26): \quad\left(x \rightarrow_{N} y\right) \rightarrow_{N}\left[\left(y \rightarrow_{N} x\right) \rightarrow_{N}\left[(z \rightarrow x) \rightarrow_{N}(z \rightarrow y)\right]\right]=1$,

$(E 27): \quad(\sim(x \rightarrow y)) \rightarrow_{N}(x \wedge \sim y)=1$, 


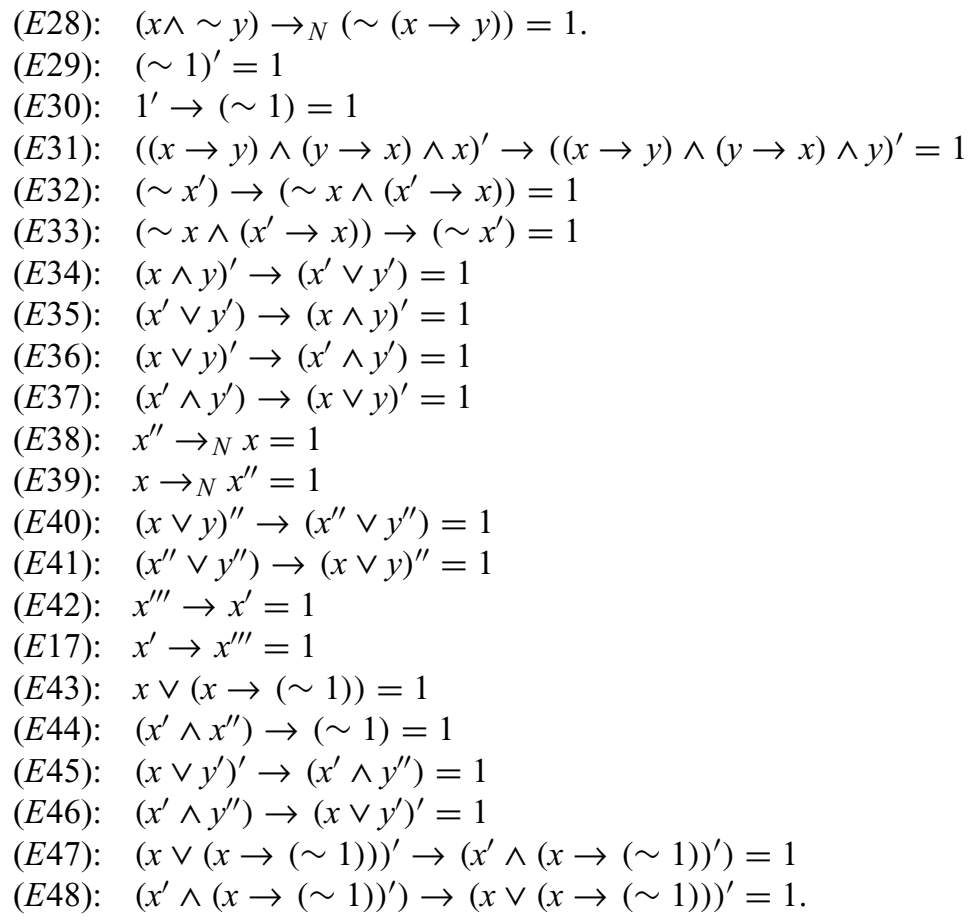

We also list the following sets formed by the above equations:

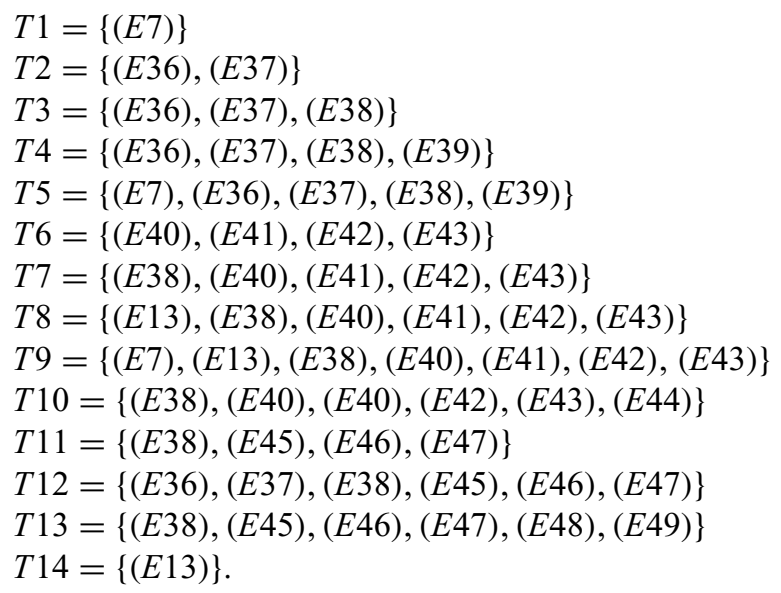

In what follows we recall some definition given in [10], which we shall use later.

\section{DEFINITION 3.1}

An algebra $(A, \wedge, \vee, \rightarrow, \sim, 1)$ of type $(2,2,2,1,0)$ is a semi-Nelson algebra if the conditions $(E 18)-$ (E28) are satisfied.

We write $\mathbb{S N}$ for the variety of semi-Nelson algebras [10]. These algebras are linked with Nelson algebras in many senses (see [10]). In particular, the variety of Nelson algebras is a proper subvariety 


\section{Dually Hemimorphic Semi-Nelson Algebras}

of the variety of semi-Nelson algebras [25]. Axioms (E18) and (E19) are those given by Sholander in [23], which define distributive lattices, so in what follows we will use freely the arithmetic rules of distributive lattices.

\section{DEFINITION 3.2}

An algebra $\left(A, \wedge, \vee, \rightarrow, \sim,{ }^{\prime}, 1\right)$ of type $(2,2,2,1,1,0)$ is a dually hemimorphic semi-Nelson algebra if $(A, \wedge, \vee, \rightarrow, \sim, 1)$ is a semi-Nelson algebra and satisfies the equations from (E29) to $(E 35)$.

We denote by $\mathbb{D H} \mathbb{M} \mathbb{S N}$ to the variety of dually hemimorphic semi-Nelson algebras.

The following lemma involves some algebraic properties of $\mathbb{D H} \mathbb{M} \mathbb{S}$.

\section{LEMMA 3.3}

Let $\left(A, \wedge, \vee, \rightarrow, \sim,{ }^{\prime}, 1\right) \in \mathbb{D} \mathbb{H M} \mathbb{S N}$ and $a, b, c \in A$. Then the following properties hold:

(1) $a \vee \sim 1=a$,

(2) $1 \rightarrow_{N} a=a$,

(3) $a \rightarrow_{N} a=1$,

(4) $a \rightarrow_{N}(b \wedge c)=\left(a \rightarrow_{N} b\right) \wedge\left(a \rightarrow_{N} c\right)$,

(5) if $a \leq b$ then $a \rightarrow_{N} b=1$,

(6) $(a \vee b) \rightarrow_{N} c=\left(a \rightarrow_{N} c\right) \wedge\left(b \rightarrow_{N} c\right)$,

(7) $a \leq b$ if and only if $a \rightarrow_{N} b=1$ and $\sim b \rightarrow_{N} \sim a=1$,

(8) if $a \rightarrow_{N} b=b \rightarrow_{N} c=1$ then $a \rightarrow_{N} c=1$,

(9) $(\sim 1) \rightarrow_{N} a=1$,

(10) $(a \wedge \sim a) \rightarrow_{N} b=1$,

(11) $a \rightarrow_{N} b=a \rightarrow_{N}(a \wedge b)$,

(12) $(a \rightarrow b) \rightarrow_{N}\left(a \rightarrow_{N} b\right)=1$,

(13) $a \rightarrow_{N} b=1$ and $b \rightarrow_{N} a=1$ if and only if $a \rightarrow b=1$ and $b \rightarrow a=1$,

(14) if $a \rightarrow_{N} b=1$ then $a \rightarrow_{N}(a \wedge b)=1$,

(15) $\left(a^{\prime} \vee b^{\prime} \vee c^{\prime}\right) \rightarrow_{N}(a \wedge b \wedge c)^{\prime}=1$,

(16) $(a \wedge b \wedge c)^{\prime} \rightarrow_{N}\left(a^{\prime} \vee b^{\prime} \vee c^{\prime}\right)=1$.

Proof. Items (1)-(13) follow from several results of [10, Lemmas 2.4, 2.6 and 2.7].

Let us prove item (13). By hypothesis we have that $a \rightarrow_{N} b=1$. Then apply item (11).

In order to check (15) note that, by (E35), we have that $\left(a^{\prime} \vee(b \wedge c)^{\prime}\right) \rightarrow(a \wedge(b \wedge c))^{\prime}=1$. Then it follows from items (2) and (12) that

$$
\left(a^{\prime} \vee(b \wedge c)^{\prime}\right) \rightarrow_{N}(a \wedge(b \wedge c))^{\prime}=1
$$

Taking into account equation (E35) we obtain

$$
\left(b^{\prime} \vee c^{\prime}\right) \rightarrow(b \wedge c)^{\prime}=1 .
$$

Hence, it follows from by items (2) and (12) that $\left(b^{\prime} \vee c^{\prime}\right) \rightarrow_{N}(b \wedge c)^{\prime}=1$. So, by (5),

$$
(b \wedge c)^{\prime} \rightarrow_{N}\left(a^{\prime} \vee(b \wedge c)^{\prime}\right)=1 .
$$

Using item (8) in equations (3.2) and (3.3) we deduce the equality

$$
\left(b^{\prime} \vee c^{\prime}\right) \rightarrow_{N}\left(a^{\prime} \vee(b \wedge c)^{\prime}\right)=1
$$


Class of algebras

Dually hemimorphic Nelson algebras

Semi Nelson algebras with an Ockham operation

Semi Nelson algebras with a dual MS-operation

De Morgan (or symmetric) semi-Nelson algebras

De Morgan (or symmetric) Nelson algebras

Semi-Nelson algebras with a dual semi-De Morgan operation

Dually quasi-De Morgan semi-Nelson algebras

Semi Nelson algebras with a dual pseudocomplementation

Nelson algebras with a dual pseudocomplementation

Boolean semi Nelson algebras with a dual quasi De Morgan operation

Semi Nelson algebras with a dual quasi Stone operation

Semi Nelson algebras with a dual Stone operation

Semi Nelson algebras with a blended dual quasi Stone operation

Semi Nelson algebras with a dual semicomplementation

\begin{tabular}{|c|c|}
\hline Def. set & Notation \\
\hline$T 1$ & $\mathbb{D H M N}$ \\
\hline$T 2$ & $\mathbb{O C K S N}$ \\
\hline$T 3$ & $\mathbb{D} m s \mathbb{S N}$ \\
\hline$T 4$ & $\mathbb{D M S N}$ \\
\hline$T 5$ & $\mathbb{D M N}$ \\
\hline$T 6$ & $\mathbb{D S D S N}$ \\
\hline$T 7$ & $\mathbb{D Q D S N}$ \\
\hline$T 8$ & $\mathbb{D P C S N}$ \\
\hline$T 9$ & $\mathbb{D P C N}$ \\
\hline$T 10$ & $\mathbb{D Q D D B S N}$ \\
\hline$T 11$ & $\mathbb{D Q S S N}$ \\
\hline$T 12$ & $\mathbb{D S S N}$ \\
\hline$T 13$ & $\mathbb{B D Q S S N}$ \\
\hline$T 14$ & $\mathbb{D S C S N}$ \\
\hline
\end{tabular}

FIGURE 2. Subvarieties of $\mathbb{D H} \mathbb{M} S \mathbb{N}$.

Therefore, $\left(a^{\prime} \vee\left(b^{\prime} \vee c^{\prime}\right)\right) \rightarrow_{N}\left(a^{\prime} \vee(b \wedge c)^{\prime}\right) \stackrel{(6)}{=}\left(a^{\prime} \rightarrow_{N}\left(a^{\prime} \vee(b \wedge c)^{\prime}\right)\right) \wedge\left(\left(b^{\prime} \vee c^{\prime}\right) \rightarrow_{N}\left(a^{\prime} \vee(b \wedge c)^{\prime}\right)\right)$ $\stackrel{(5)}{=} 1 \wedge\left(\left(b^{\prime} \vee c^{\prime}\right) \rightarrow_{N}\left(a^{\prime} \vee(b \wedge c)^{\prime}\right)\right)=\left(b^{\prime} \vee c^{\prime}\right) \rightarrow_{N}\left(a^{\prime} \vee(b \wedge c)^{\prime}\right) \stackrel{(3.4)}{=} 1$. Then using item (8) and the equation (3.1), $\left(a^{\prime} \vee\left(b^{\prime} \vee c^{\prime}\right)\right) \rightarrow_{N}(a \wedge(b \wedge c))^{\prime}=1$.

The proof of item (16) is similar by using identity (E34).

Again, to avoid confusions, we present the definitions of subvarieties of $\mathbb{D H M} \mathbb{M}$ by using a table.

\section{DEFINITION 3.4}

The Figure 2 is the definition of subvarieties of $\mathbb{D H M} \mathbb{M N}$, where the first column indicates the name of the class, the second one indicates the defining set of equations modulo $\mathbb{D H M} \mathbb{M N}$ and the third one indicates its notation along this paper.

In what follows we will show some examples of algebras of $\mathbb{D H} \mathbb{M} \mathbb{S}$.

\section{L1}

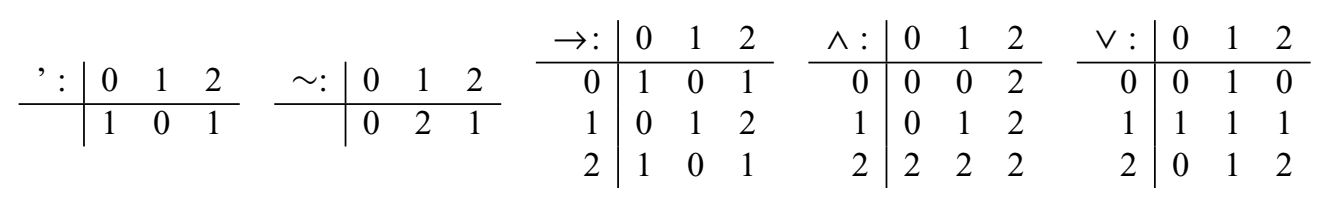

We have that $\mathbf{L} 1 \in \mathbb{D M S N} \cap \mathbb{D P C S N} \cap \mathbb{D Q D S N}$ and $\mathbf{L} 1 \notin \mathbb{D M N} \cup \mathbb{D P C N} \cup \mathbb{D Q D B S N} \cup$ $\mathbb{D H M N}$.

L2

\begin{tabular}{|c|c|c|c|c|c|c|c|c|c|c|c|c|c|c|c|c|c|}
\hline ' : & 0 & 1 & 2 & 3 & 4 & $\sim:$ & 0 & 1 & $?$ & 3 & & & & & & & \\
\hline & 1 & 4 & 1 & 1 & 1 & & & 3 & ) & 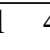 & & & & & & & \\
\hline$\rightarrow:$ & 0 & 1 & 2 & 3 & 4 & $\wedge:$ & 0 & 1 & 2 & 3 & 4 & $\vee:$ & 0 & 1 & 2 & 3 & 4 \\
\hline 0 & 1 & 0 & 2 & 2 & 4 & 0 & 0 & 0 & 2 & 3 & 4 & 0 & 0 & 1 & 0 & 0 & 0 \\
\hline 1 & 0 & 1 & 2 & 3 & 4 & 1 & 0 & 1 & 2 & 3 & 4 & 1 & 1 & 1 & 1 & 1 & 1 \\
\hline 2 & 0 & 0 & 1 & 1 & 1 & 2 & 2 & 2 & 2 & 3 & 2 & 2 & 0 & 1 & 2 & 2 & 4 \\
\hline 3 & 0 & 0 & 1 & 1 & 1 & 3 & 3 & 3 & 3 & 3 & 3 & 3 & 0 & 1 & 2 & 3 & 4 \\
\hline 4 & 0 & 0 & 1 & 1 & 1 & 4 & 4 & 4 & 2 & 3 & 4 & 4 & 0 & 1 & 4 & 4 & 4 \\
\hline
\end{tabular}


In this example we have that $\mathbf{L} 2 \in \mathbb{D} m s \mathbb{S N}$ and $\mathbf{L} 2 \notin \mathbb{D M S N}$.

L3

\begin{tabular}{|c|c|c|c|c|c|c|c|c|c|c|c|c|c|c|c|c|c|}
\hline : & 0 & 1 & 2 & 3 & 4 & $\sim:$ & 0 & & & & & & & & & & \\
\hline & 1 & 4 & 1 & 1 & 1 & & & 3 &  & 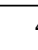 & & & & & & & \\
\hline$\rightarrow:$ & 0 & 1 & 2 & 3 & 4 & $\wedge:$ & 0 & 1 & 2 & 3 & 4 & $\vee:$ & 0 & 1 & 2 & 3 & 4 \\
\hline 0 & 1 & 1 & 2 & 2 & 4 & 0 & 0 & 0 & 2 & 3 & 4 & 0 & 0 & 1 & 0 & 0 & 0 \\
\hline 1 & 0 & 1 & 2 & 3 & 4 & 1 & 0 & 1 & 2 & 3 & 4 & 1 & 1 & 1 & 1 & 1 & 1 \\
\hline 2 & 1 & 1 & 1 & 1 & 1 & 2 & 2 & 2 & 2 & 3 & 2 & 2 & 0 & 1 & 2 & 2 & 4 \\
\hline 3 & 1 & 1 & 1 & 1 & 1 & 3 & 3 & 3 & 3 & 3 & 3 & 3 & 0 & 1 & 2 & 3 & 4 \\
\hline 4 & 1 & 1 & 1 & 1 & 1 & 4 & 4 & 4 & 2 & 3 & 4 & 4 & 0 & 1 & 4 & 4 & 4 \\
\hline
\end{tabular}

We have that $\mathbf{L} 3 \in \mathbb{D H} \mathbb{M N}$ and $\mathbf{L} 3 \notin \mathbb{D M N}$.

L4

\begin{tabular}{|c|c|c|c|c|c|c|c|c|c|c|c|c|c|c|c|c|c|}
\hline . & 0 & 1 & 2 & 3 & & $\sim:$ & 0 & 1 & & & & & & & & & \\
\hline & 2 & 2 & 1 & 1 & 1 & & 3 & 4 & & & & & & & & & \\
\hline$\rightarrow:$ & 0 & 1 & 2 & 3 & 4 & $\wedge:$ & 0 & 1 & 2 & 3 & 4 & $\vee:$ & 0 & 1 & 2 & 3 & 4 \\
\hline 0 & 1 & 0 & 2 & 3 & 3 & 0 & 0 & 0 & 2 & 3 & 4 & 0 & 0 & 1 & 0 & 0 & 0 \\
\hline 1 & 0 & 1 & 2 & 3 & 4 & 1 & 0 & 1 & 2 & 3 & 4 & 1 & 1 & 1 & 1 & 1 & 1 \\
\hline 2 & 0 & 0 & 1 & 1 & 1 & 2 & 2 & 2 & 2 & 3 & 4 & 2 & 0 & 1 & 2 & 2 & 2 \\
\hline 3 & 0 & 0 & 1 & 1 & 1 & 3 & 3 & 3 & 3 & 3 & 4 & 3 & 0 & 1 & 2 & 3 & 3 \\
\hline 4 & 0 & 0 & 1 & 1 & 1 & 4 & 4 & 4 & 4 & 4 & 4 & 4 & 0 & 1 & 2 & 3 & 4 \\
\hline
\end{tabular}

We have that $\mathbf{L} 4 \in \mathbb{O} \mathbb{C} \mathbb{S N} \cap \mathbb{D S D S N}$ and $\mathbf{L} 4 \notin \mathbb{D} m s \mathbb{N} \cup \mathbb{D Q D S N}$.

L5

\begin{tabular}{|c|c|c|c|c|c|c|c|c|c|c|c|c|c|c|c|c|c|}
\hline ': & 0 & 1 & 2 & 3 & & $\sim:$ & 0 & 1 & $?$ & & & & & & & & \\
\hline & 0 & 4 & 1 & 1 & 1 & & & 3 & ) & & & & & & & & \\
\hline$\rightarrow:$ & 0 & 1 & 2 & 3 & 4 & $\wedge:$ & 0 & 1 & 2 & 3 & 4 & $\vee:$ & 0 & 1 & 2 & 3 & 4 \\
\hline 0 & 1 & 0 & 2 & 2 & 4 & 0 & 0 & 0 & 2 & 3 & 4 & 0 & 0 & 1 & 0 & 0 & 0 \\
\hline 1 & 0 & 1 & 2 & 3 & 4 & 1 & 0 & 1 & 2 & 3 & 4 & 1 & 1 & 1 & 1 & 1 & 1 \\
\hline 2 & 0 & 0 & 1 & 1 & 1 & 2 & 2 & 2 & 2 & 3 & 2 & 2 & 0 & 1 & 2 & 2 & 4 \\
\hline 3 & 0 & 0 & 1 & 1 & 1 & 3 & 3 & 3 & 3 & 3 & 3 & 3 & 0 & 1 & 2 & 3 & 4 \\
\hline 4 & 0 & 0 & 1 & 1 & 1 & 4 & 4 & 4 & 2 & 3 & 4 & 4 & 0 & 1 & 4 & 4 & \\
\hline
\end{tabular}

We have that $\mathbf{L} 5 \in \mathbb{D Q D S N}$ and $\mathbf{L} 5 \notin \mathbb{D P C S N} \cup \mathbb{D S C S N}$.

L6

\begin{tabular}{|c|c|c|c|c|c|c|c|c|c|c|c|c|c|c|c|c|c|}
\hline$:$ & $\begin{array}{ll}0 \\
\end{array}$ & 12 & 2 & 3 & 4 & $\sim:$ & 0 & 1 & $?$ & & & & & & & & \\
\hline & 0 & 41 & 1 & 1 & 1 & & & 3 & ) & & 4 & & & & & & \\
\hline$\rightarrow:$ & 0 & 1 & 2 & 3 & 4 & $\wedge:$ & 0 & 1 & 2 & 3 & 4 & $\vee:$ & 0 & 1 & 2 & 3 & 4 \\
\hline 0 & 1 & 1 & 2 & 2 & 4 & 0 & 0 & 0 & 2 & 3 & 4 & 0 & 0 & 1 & 0 & 0 & 0 \\
\hline 1 & 0 & 1 & 2 & 3 & 4 & 1 & 0 & 1 & 2 & 3 & 4 & 1 & 1 & 1 & 1 & 1 & 1 \\
\hline 2 & 1 & 1 & 1 & 1 & 1 & 2 & 2 & 2 & 2 & 3 & 2 & 2 & 0 & 1 & 2 & 2 & 4 \\
\hline 3 & 1 & 1 & 1 & 1 & 1 & 3 & 3 & 3 & 3 & 3 & 3 & 3 & 0 & 1 & 2 & 3 & 4 \\
\hline 4 & 1 & 1 & 1 & 1 & 1 & 4 & 4 & 4 & 2 & 3 & 4 & 4 & 0 & 1 & 4 & 4 & 4 \\
\hline
\end{tabular}

We have that $\mathbf{L} 6 \in \mathbb{D} \mathbb{H} \mathbb{M}$ and $\mathbf{L} 6 \notin \mathbb{D P C N}$. 
L7



We have that $\mathbf{L} 7 \in \mathbb{D H M S N}$ and $\mathbf{L} 7 \notin \mathbb{D S D S N}$.

\section{Relation between $\mathbb{D H M} \mathbb{M} H$ and $\mathbb{D H M} \mathbb{M}$}

In this section we describe the constructions that realizes the connection between dually hemimorphic semi-Nelson algebras and dually hemimorphic semi-Heyting algebras.

In order to avoid confusions, in this sections we will use the following notation:

\begin{tabular}{c|c} 
Class of algebras & Language \\
\hline $\mathbb{D H} \mathbb{H} \mathbb{S N}$ & $\left\{\wedge, \vee, \rightarrow, \sim,^{\prime}, 1\right\}$ \\
$\mathbb{D H} \mathbb{M} \mathbb{S H}$ & $\left\{\cap, \cup, \Rightarrow,^{\dagger}, \perp, \top\right\}$
\end{tabular}

\subsection{The quotient algebra}

In what follows we will prove that for every dually hemimorphic semi-Nelson algebra it is possible to build up a dually hemimorphic semi-Nelson algebra.

We start with some preliminary definitions and results.

Let $\mathbf{A}$ be a semi-Nelson algebra. It follows from [10, Lemma 3.1] that the binary relation $\equiv$ defined on $A$ by

$$
x \equiv y \text { if and only if } x \rightarrow y=1 \text { and } y \rightarrow x=1
$$

is an equivalence relation compatible with the operations $\sim, \wedge, \vee$ and $\rightarrow$.

For every $x \in A$ we write $\llbracket x \rrbracket$ for the equivalence class associated with $x$.

Note that it follows from item (13) of Lemma 3.1 that the relation $\equiv$ can be replaced by

$$
x \equiv y \text { if and only if } x \rightarrow_{N} y=1 \text { and } y \rightarrow_{N} x=1 .
$$


Let $\mathbf{A} \in \mathbb{S N}$. We denote by $\mathbf{s H}(\mathbf{A})$ to the algebra $(A / \equiv, \cap, \cup, \Rightarrow, \perp, \top)$, where $A / \equiv$ is the set of equivalence classes and the operations on $A / \equiv$ are defined as follows:

- $\perp=\llbracket \sim 1 \rrbracket$,

- $\mathrm{\top}=\llbracket 1 \rrbracket$

- $\llbracket x \rrbracket \cap \llbracket y \rrbracket=\llbracket x \wedge y \rrbracket$,

- $\llbracket x \rrbracket \cup \llbracket y \rrbracket=\llbracket x \vee y \rrbracket$,

- $\llbracket x \rrbracket \Rightarrow \llbracket y \rrbracket=\llbracket x \rightarrow y \rrbracket$.

By [10, Theorem 3.4] we have that $\mathbf{s H ( A )}$ is a semi-Heyting algebra.

\section{LEMMA 4.1}

Let $\mathbf{A}=\left(A, \wedge, \vee, \rightarrow, \sim,{ }^{\prime}, 1\right) \in \mathbb{D H} \mathbb{M} \mathbb{S}$. The relation $\equiv$ is compatible with the operation '.

Proof. Let us consider $x, y \in A$ such that $x \equiv y$. Then $x \rightarrow y=y \rightarrow x=1$. Hence,

$1 \stackrel{(E 31)}{=}((x \rightarrow y) \wedge(y \rightarrow x) \wedge x)^{\prime} \rightarrow((x \rightarrow y) \wedge(y \rightarrow x) \wedge y)^{\prime}=(1 \wedge 1 \wedge x)^{\prime} \rightarrow(1 \wedge 1 \wedge y)^{\prime}$ $=x^{\prime} \rightarrow y^{\prime}$.

Similarly, it can be verified that $y^{\prime} \rightarrow x^{\prime}=1$. Therefore, $x^{\prime} \equiv y^{\prime}$.

Let $\mathbf{A} \in \mathbb{D H} M \mathbb{M N}$. We also denote by $\mathbf{s H}(\mathbf{A})$ the algebra $\left(A / \equiv, \cap, \cup, \Rightarrow,{ }^{\dagger}, \perp, \top\right)$, where the operation ${ }^{\dagger}$ is defined by

- $\llbracket x \rrbracket^{\dagger}=\llbracket x^{\prime} \rrbracket$.

The well definition of the previous operation follows from Lemma 4.1.

THEOREM 4.2

Let $\mathbf{A} \in \mathbb{D H} M \mathbb{S N}$. Then $\mathbf{s H}(\mathbf{A})$ is a dually hemimorphic semi-Heyting algebra.

Proof. We will show that $\mathbf{s H}(\mathbf{A})$ satisfies the equations (E4), (E5) and (E6).

It follows from (E29) that $\perp^{\dagger}=\llbracket \sim 1 \rrbracket^{\dagger}=\llbracket(\sim 1)^{\prime} \rrbracket=\llbracket 1 \rrbracket=T$, so (E4) is satisfied.

Now we will prove (E5). Taking into account (9) of Lemma 3.3 we have that

$$
(\sim 1) \rightarrow_{N} 1^{\prime}=1 .
$$

By (E30) it holds that $1^{\prime} \rightarrow(\sim 1)=1$. Hence, it follows from (2) and (12) of Lemma 3.3 that

$$
1^{\prime} \rightarrow_{N}(\sim 1)=1 \text {. }
$$

Applying (13) of Lemma 3.3 in (4.1) and (4.2) we obtain that $(\sim 1) \rightarrow 1^{\prime}=1^{\prime} \rightarrow(\sim 1)=1$. Thus,

$$
\top^{\dagger}=\llbracket 1 \rrbracket^{\dagger}=\llbracket 1^{\prime} \rrbracket=\llbracket \sim 1 \rrbracket=\perp .
$$

Straightforward computations based on (E34) and (E34) prove that $\mathbf{s H}(\mathbf{A})$ satisfies (E6).

LEMMA 4.3

Let $\mathbf{A} \in \mathbb{D H} \mathbb{M} \mathbb{S}$. The following conditions are satisfied:

1. If $\mathbf{A}$ satisfies (E7) then $\mathbf{s H}(\mathbf{A})$ satisfies $(E 7)$.

2. If $\mathbf{A}$ satisfies (E38) then $\mathbf{~} \mathbf{H}(\mathbf{A})$ satisfies $(E 9)$.

3. If A satisfies (E45) then $\mathbf{s H}(\mathbf{A})$ satisfies $(E 17)$.

Proof. Let $x, y \in A$.

1. $(\llbracket x \rrbracket \cap \llbracket y \rrbracket) \Rightarrow \llbracket x \rrbracket=\llbracket x \wedge y \rrbracket \Rightarrow \llbracket x \rrbracket=\llbracket(x \wedge y) \rightarrow x \rrbracket \stackrel{(E 7)}{=} \llbracket 1 \rrbracket=\top$. 
2. It follows from (E38) that $x^{\prime \prime} \rightarrow_{N} x=1$. Then, by (14) of Lemma 3.3, $x^{\prime \prime} \rightarrow_{N}\left(x^{\prime \prime} \wedge x\right)=1$. Besides, by (5) of Lemma 3.3 we have that $\left(x^{\prime \prime} \wedge x\right) \rightarrow_{N} x^{\prime \prime}=1$. Hence, $\llbracket x^{\prime \prime} \rrbracket=\llbracket x^{\prime \prime} \wedge x \rrbracket$.

3. It follows from $(E 45)$ that $\left(x^{\prime} \wedge x^{\prime \prime}\right) \rightarrow_{N}(\sim 1)=\left(x^{\prime} \wedge x^{\prime \prime}\right) \rightarrow(\sim 1)=1$. By (9) of Lemma 3.3 we conclude that $(\sim 1) \rightarrow_{N}\left(x^{\prime} \wedge x^{\prime \prime}\right)=1$.

Let $M_{1}$ be the set formed by the following elements:

- $\{\mathbb{D H M} \mathbb{M}, \mathbb{D H} \mathbb{M} \mathbb{N}\}$,

- $\{\mathbb{O C K S H}, \mathbb{O C K \mathbb { N }}\}$,

- $\{\mathbb{D} m s \mathbb{S H}, \mathbb{D} m s \mathbb{S N}\}$,

- $\{\mathbb{D M} \mathbb{M} H, \mathbb{D M} \mathbb{S}\}$,

- $\{\mathbb{D M} \mathbb{M}, \mathbb{D M N}\}$,

- $\{\mathbb{D S D S H}, \mathbb{D S D S N}\}$,

- $\{\mathbb{D Q D S H}, \mathbb{D Q D S N}\}$,

- $\{\mathbb{D P C S H}, \mathbb{D P C S N}\}$,

- $\{\mathbb{D P C H}, \mathbb{D P C N}$,

- $\{\mathbb{D Q D B S H}, \mathbb{D Q D D B S N}\}$,

- $\{\mathbb{D Q S S H}, \mathbb{D Q S S N}\}$,

- $\{\mathbb{D S S H}, \mathbb{D S S N}\}$,

- $\{\mathbb{B D Q S S H}, \mathbb{B D Q S S N}\}$,

- $\{\mathbb{D S C S H I}, \mathbb{D S C S N}$.

\section{THEOREM 4.4}

Let $\{\mathbb{A}, \mathbb{B}\} \in M_{1}$ and $\mathbf{B}=\left(B, \wedge, \vee, \sim,{ }^{\prime}, 1\right) \in \mathbb{B}$. Then $\mathbf{s H}(\mathbf{B}) \in \mathbb{A}$.

PROOF. It follows from straightforward computations based on Theorem 4.2 and Lemma 4.3.

\subsection{Vakarelov's construction}

We will see that Vakarelov's construction on semi Heyting algebras works as well for dually hemimorphic semi-Heyting algebras [10].

We start with some preliminary definitions and results.

Let $\mathbf{A}=(A, \cap, \cup, \Rightarrow, \perp, \top) \in \mathbb{S H}$. Define the following set:

$$
\mathrm{K}(A)=\{(a, b) \in A \times A: a \cap b=0\} .
$$

We denote by $\mathbf{V}_{k}(\mathbf{A})$ to the algebra $(\mathrm{K}(A), \wedge, \vee, \rightarrow, \sim, 1)$, where the operations are given by

$(V 1) \quad(a, b) \wedge(c, d)=(a \cap c, b \cup d)$,

(V2) $(a, b) \vee(c, d)=(a \cup c, b \cap d)$,

$(V 3) \quad(a, b) \rightarrow(c, d)=(a \Rightarrow c, a \cap d)$,

$(V 4) \sim(a, b)=(b, a)$,

(V5) $1=(\top, \perp)$.

It follows from [10, Theorem 4.1] that if $\mathbf{A}=(A, \cap, \cup, \Rightarrow, \perp, \top) \in \mathbb{S H}$, then $\mathbf{V}_{k}(\mathbf{A}) \in \mathbb{S N}$.

\section{DEFINITION 4.5}

Let $\mathbf{A}=\left(A, \cap, \cup, \Rightarrow,^{\dagger}, \perp, \top\right)$ be a dually hemimorphic semi-Heyting algebra. For $(a, b) \in \mathrm{K}(A)$ we 
Dually Hemimorphic Semi-Nelson Algebras

define the following unary operation on $\mathrm{K}(A)$ :

$$
(a, b)^{\prime}=\left(a^{\dagger}, b \cap\left(a^{\dagger} \Rightarrow a\right)\right) .
$$

Note that ' is well defined. In fact, if $a \cap b=0$ then $a^{\dagger} \cap b \cap\left(a^{\dagger} \Rightarrow a\right) \stackrel{(E 1)}{=} a^{\dagger} \cap b \cap a=0$.

For $\mathbf{A} \in \mathbb{D H} \mathbb{M} \mathbb{S H}$ we also write $\mathbf{V}_{k}(\mathbf{A})$ for the algebra $\left(\mathrm{K}(A), \wedge, \vee, \rightarrow, \sim,{ }^{\prime}, 1\right)$.

THEOREM 4.6

If $\mathbf{A}=\left(A, \cap, \cup, \Rightarrow,{ }^{\dagger}, \perp, \top\right) \in \mathbb{D H M} \mathbb{M} H$, then $\mathbf{V}_{k}(\mathbf{A}) \in \mathbb{D H M} \mathbb{M}$.

Proof. We will see that $\mathbf{V}_{k}$ (A) satisfies the equations (E29)-(E35). Let $(a, b),(c, d) \in \mathrm{K}(A)$. Then

(E29): $\quad(\sim 1)^{\prime}=(\sim(\top, \perp))^{\prime}=(\perp, \top)^{\prime}=\left(\perp^{\dagger}, \top \cap\left(\perp^{\dagger} \Rightarrow \perp\right)\right)=\left(\perp^{\dagger}, \perp^{\dagger} \Rightarrow \perp\right) \stackrel{(E 4)}{=}(\top, \top \Rightarrow$ $\perp)=(\top, \perp)=1$.

(E30): $\quad 1^{\prime} \rightarrow(\sim 1)=(\top, \perp)^{\prime} \rightarrow(\sim(\top, \perp))=\left(\mathrm{T}^{\dagger}, \perp \cap\left(\mathrm{T}^{\dagger} \rightarrow \mathrm{T}\right)\right) \rightarrow(\perp, \mathrm{\top})=\left(\mathrm{T}^{\dagger}, \perp\right) \rightarrow$ $(\perp, \top) \stackrel{(E 5)}{=}(\perp, \perp) \rightarrow(\perp, \top)=(\perp \Rightarrow \perp, \perp \cap \top)=(\top, \perp)=1$.

(E31): First, notice that

$$
\begin{aligned}
((a, b) \rightarrow(c, d)) \wedge((c, d) \rightarrow(a, b)) & =(a \Rightarrow c, a \cap d) \wedge(c \Rightarrow a, c \cap b) \\
& =((a \Rightarrow c) \cap(c \Rightarrow a)),(a \cap d) \cup(c \cap b)) .
\end{aligned}
$$

Then $((a, b) \rightarrow(c, d)) \wedge((c, d) \rightarrow(a, b)) \wedge(a, b) \stackrel{(4.3)}{=}((a \Rightarrow c) \cap(c \Rightarrow a)),(a \cap d) \cup(c \cap$ $b)) \wedge(a, b)=((a \Rightarrow c) \cap(c \Rightarrow a) \cap a,(a \cap d) \cup(c \cap b) \cup b)=((a \Rightarrow c) \cap(c \Rightarrow a) \cap a,(a \cap d) \cup b)$ $\stackrel{(E 1)}{=}(a \cap c,(a \cap d) \cup b)$.

In consequence,

$$
((a, b) \rightarrow(c, d)) \wedge((c, d) \rightarrow(a, b)) \wedge(a, b)=(a \cap c,(a \cap d) \cup b) .
$$

Similarly, we have that

$$
((a, b) \rightarrow(c, d)) \wedge((c, d) \rightarrow(a, b)) \wedge(c, d)=(a \cap c,(c \cap b) \cup d) .
$$

Thus,

$(((a, b) \rightarrow(c, d)) \wedge((c, d) \rightarrow(a, b)) \wedge(a, b))^{\prime} \rightarrow(((a, b) \rightarrow(c, d)) \wedge((c, d) \rightarrow(a, b)) \wedge$ $(c, d))^{\prime} \stackrel{(4.4) \text { and }(4.5)}{=}(a \cap c,(a \cap d) \cup b)^{\prime} \rightarrow(a \cap c,(c \cap b) \cup d)^{\prime}=\left((a \cap c)^{\dagger},((a \cap d) \cup b) \cap((a \cap\right.$ $\left.\left.c)^{\dagger} \rightarrow(a \cap c)\right)\right) \rightarrow\left((a \cap c)^{\dagger},((c \cap b) \cup d) \cap\left((a \cap c)^{\dagger} \rightarrow(a \cap c)\right)\right)=\left((a \cap c)^{\dagger} \Rightarrow(a \cap c)^{\dagger},(a \cap\right.$ $\left.c)^{\dagger} \cap((c \cap b) \cup d) \cap\left((a \cap c)^{\dagger} \rightarrow(a \cap c)\right)\right)=\left(\top,(a \cap c)^{\dagger} \cap((c \cap b) \cup d) \cap\left((a \cap c)^{\dagger} \rightarrow(a \cap c)\right)\right)$ $\stackrel{(E 1)}{=}\left(\top,(a \cap c)^{\dagger} \cap((c \cap b) \cup d) \cap(a \cap c)\right)=(\top, \perp)=1$.

(E32): First, note that

$$
\begin{aligned}
\sim(a, b)^{\prime} & =\sim\left(a^{\dagger}, b \cap\left(a^{\dagger} \Rightarrow a\right)\right) \\
& =\left(b \cap\left(a^{\dagger} \Rightarrow a\right), a^{\dagger}\right) .
\end{aligned}
$$

and

$$
\begin{aligned}
\left(\sim(a, b) \wedge\left((a, b)^{\prime} \rightarrow(a, b)\right)\right) & =\left(\sim(a, b) \wedge\left(\left(a^{\dagger}, b \cap\left(a^{\dagger} \Rightarrow a\right)\right) \rightarrow(a, b)\right)\right) \\
& =\left((b, a) \wedge\left(\left(a^{\dagger}, b \cap\left(a^{\dagger} \Rightarrow a\right)\right) \rightarrow(a, b)\right)\right) \\
& =\left(b \cap\left(a^{\dagger} \Rightarrow a\right), a \cup\left(a^{\dagger} \cap b\right)\right) .
\end{aligned}
$$

Hence, 


$$
\begin{aligned}
& \left(\sim(a, b)^{\prime}\right) \rightarrow\left(\sim(a, b) \wedge\left((a, b)^{\prime} \rightarrow(a, b)\right)\right) \stackrel{(4.6) \text { and }(4.7)}{=}\left(b \cap\left(a^{\dagger} \Rightarrow a\right), a^{\dagger}\right) \rightarrow\left(b \cap \left(a^{\dagger} \Rightarrow\right.\right. \\
& \left.a), a \cup\left(a^{\dagger} \cap b\right)\right)=\left(\top, b \cap\left(a^{\dagger} \Rightarrow a\right) \cap\left(a \cup\left(a^{\dagger} \cap b\right)\right)\right)=\left(\top,\left(b \cap\left(a^{\dagger} \Rightarrow a\right) \cap a\right) \cup\left(b \cap \left(a^{\dagger} \Rightarrow\right.\right.\right. \\
& \left.\left.a) \cap a^{\dagger} \cap b\right)\right)=\left(\top, \perp \cup\left(b \cap\left(a^{\dagger} \Rightarrow a\right) \cap a^{\dagger} \cap b\right)\right)=\left(\top, b \cap\left(a^{\dagger} \Rightarrow a\right) \cap a^{\dagger} \cap b\right) \stackrel{(E 1)}{=}\left(\top, b \cap a^{\dagger} \cap a\right) \\
& =(\top, \perp)=1 . \\
(\mathrm{E} 33): & \left(\sim(a, b) \wedge\left((a, b)^{\prime} \rightarrow(a, b)\right)\right) \rightarrow\left(\sim(a, b)^{\prime}\right)\left(\sim(a, b) \wedge\left((a, b)^{\prime} \rightarrow(a, b)\right)\right) \rightarrow\left(\sim(a, b)^{\prime}\right) \\
& (4.6) \text { and }(4.7) \\
& \left.(b 1)=\left(a^{\dagger} \Rightarrow a\right), a \cup\left(a^{\dagger} \cap b\right)\right) \rightarrow\left(b \cap\left(a^{\dagger} \Rightarrow a\right), a^{\dagger}\right)=\left(\top, b \cap\left(a^{\dagger} \Rightarrow a\right) \cap a^{\dagger}\right) \\
& \stackrel{(}{=}\left(\top, b \cap a^{\dagger} \cap a\right)=(\top, \perp)=1 .
\end{aligned}
$$

(E34): We have that

$$
\begin{aligned}
((a, b) \wedge(c, d))^{\prime} & =(a \cap c, b \cup d)^{\prime} \\
& =\left((a \cap c)^{\dagger},(b \cup d) \cap\left((a \cap c)^{\dagger} \Rightarrow(a \cap c)\right)\right) .
\end{aligned}
$$

Besides,

$$
\begin{aligned}
(a, b)^{\prime} \vee(c, d)^{\prime} & =\left(a^{\dagger}, b \cap\left(a^{\dagger} \Rightarrow a\right)\right) \vee\left(c^{\dagger}, d \cap\left(c^{\dagger} \Rightarrow c\right)\right) \\
& =\left(a^{\dagger} \cup c^{\dagger}, b \cap\left(a^{\dagger} \Rightarrow a\right) \cap d \cap\left(c^{\dagger} \Rightarrow c\right)\right)
\end{aligned}
$$

Hence,

$$
\begin{aligned}
& ((a, b) \wedge(c, d))^{\prime} \rightarrow\left((a, b)^{\prime} \vee(c, d)^{\prime}\right) \stackrel{(4.8) \text { and }(4.9)}{=}\left((a \cap c)^{\dagger},(b \cup d) \cap\left((a \cap c)^{\dagger} \Rightarrow(a \cap c)\right)\right) \rightarrow \\
& \left(a^{\dagger} \cup c^{\dagger}, b \cap\left(a^{\dagger} \Rightarrow a\right) \cap d \cap\left(c^{\dagger} \Rightarrow c\right)\right) \stackrel{(E 6)}{=}\left(a^{\dagger} \cup c^{\dagger},(b \cup d) \cap\left((a \cap c)^{\dagger} \Rightarrow(a \cap c)\right)\right) \rightarrow \\
& \left(a^{\dagger} \cup c^{\dagger}, b \cap\left(a^{\dagger} \Rightarrow a\right) \cap d \cap\left(c^{\dagger} \Rightarrow c\right)\right)=\left(\top,\left(a^{\dagger} \cup c^{\dagger}\right) \cap b \cap\left(a^{\dagger} \Rightarrow a\right) \cap d \cap\left(c^{\dagger} \Rightarrow c\right)\right) \\
& =\left(\top,\left(a^{\dagger} \cap b \cap\left(a^{\dagger} \Rightarrow a\right) \cap d \cap\left(c^{\dagger} \Rightarrow c\right)\right) \cup\left(c^{\dagger} \cap b \cap\left(a^{\dagger} \Rightarrow a\right) \cap d \cap\left(c^{\dagger} \Rightarrow c\right)\right)\right) \\
& \stackrel{(E 1)}{=}\left(\top,\left(a^{\dagger} \cap b \cap a \cap d \cap\left(c^{\dagger} \Rightarrow c\right)\right) \cup\left(c^{\dagger} \cap b \cap\left(a^{\dagger} \Rightarrow a\right) \cap d \cap\left(c^{\dagger} \Rightarrow c\right)\right)\right) \stackrel{a \cap b=\perp}{=} \\
& \left(\top, \perp \cup\left(c^{\dagger} \cap b \cap\left(a^{\dagger} \Rightarrow a\right) \cap d \cap\left(c^{\dagger} \Rightarrow c\right)\right)\right) \stackrel{(E 1)}{=}\left(\top, \perp \cup\left(c^{\dagger} \cap b \cap\left(a^{\dagger} \Rightarrow a\right) \cap d \cap c\right)\right) \\
& \stackrel{c \cap d=\perp}{=}(\top, \perp \cup \perp)=1 \text {. }
\end{aligned}
$$

Now we give some additional properties involving the subvarieties of dually hemimorphic semiHeyting algebras and dually hemimorphic semi-Nelson algebras. Since the results given here are very technical, we recommend to the reader don't read the proofs of them in a first lecture of the present paper.

\section{THEOREM 4.7}

Let $\{\mathbb{A}, \mathbb{B}\} \in M_{1}$ and $\mathbf{A}=\left(A, \cap, \cup, \Rightarrow,{ }^{\dagger}, \perp, \top\right) \in \mathbb{A}$. Then $\mathbf{V}_{k}(\mathbf{A}) \in \mathbb{B}$.

Proof. By Theorem 4.6 we know that $\mathbf{V}_{k}(\mathbf{A}) \in \mathbb{D} \mathbb{H M S N}$. Let $(a, b),(c, d) \in \mathrm{K}(A)$. Note that

$$
(a, b)^{\prime \prime}=\left(a^{\dagger}, b \cap\left(a^{\dagger} \Rightarrow a\right)\right)^{\prime}=\left(a^{\dagger \dagger}, b \cap\left(a^{\dagger} \Rightarrow a\right) \cap\left(a^{\dagger \dagger} \Rightarrow a^{\dagger}\right)\right) .
$$

We consider the following cases:

1. $\mathbb{A}=\mathbb{D} \mathbb{H} \mathbb{M}:((a, b) \wedge(c, d)) \rightarrow(a, b)=(a \cap c, b \cup d) \rightarrow(a, b)=((a \cap c) \Rightarrow a, a \cap c \cap b)=$ $((a \cap c) \Rightarrow a, \perp) \stackrel{(E 7)}{=}(\top, \perp)=1$. 
2. $\mathbb{A}=\mathbb{O} \mathbb{C} \mathbb{K} \mathbb{S H}:((a, b) \vee(c, d))^{\prime} \rightarrow\left((a, b)^{\prime} \wedge(c, d)^{\prime}\right)=(a \cup c, b \cap d)^{\prime} \rightarrow\left(\left(a^{\dagger}, b \cap\left(a^{\dagger} \Rightarrow\right.\right.\right.$ $\left.a)) \wedge\left(c^{\dagger}, d \cap\left(c^{\dagger} \Rightarrow c\right)\right)\right)=\left((a \cup c)^{\dagger},(b \cap d) \cap\left((a \cup c)^{\dagger} \Rightarrow(a \cup c)\right)\right) \rightarrow\left(a^{\dagger} \cap c^{\dagger},(b \cap\right.$ $\left.\left.\left(a^{\dagger} \Rightarrow a\right)\right) \cup\left(d \cap\left(c^{\dagger} \Rightarrow c\right)\right)\right)=\left((a \cup c)^{\dagger} \Rightarrow\left(a^{\dagger} \cap c^{\dagger}\right),(a \cup c)^{\dagger} \cap\left(\left(b \cap\left(a^{\dagger} \Rightarrow a\right)\right) \cup(d \cap\right.\right.$ $\left.\left.\left.\left(c^{\dagger} \Rightarrow c\right)\right)\right)\right) \stackrel{(E 8)}{=}\left(\left(a^{\dagger} \cap c^{\dagger}\right) \Rightarrow\left(a^{\dagger} \cap c^{\dagger}\right),\left(a^{\dagger} \cap c^{\dagger}\right) \cap\left(\left(b \cap\left(a^{\dagger} \Rightarrow a\right)\right) \cup\left(d \cap\left(c^{\dagger} \Rightarrow c\right)\right)\right)\right) \stackrel{(E 3)}{=}$ $\left(\top,\left(a^{\dagger} \cap c^{\dagger}\right) \cap\left(\left(b \cap\left(a^{\dagger} \Rightarrow a\right)\right) \cup\left(d \cap\left(c^{\dagger} \Rightarrow c\right)\right)\right)\right)=\left(\top,\left(a^{\dagger} \cap c^{\dagger} \cap b \cap\left(a^{\dagger} \Rightarrow a\right)\right) \cup\left(a^{\dagger} \cap\right.\right.$ $\left.\left.c^{\dagger} \cap d \cap\left(c^{\dagger} \Rightarrow c\right)\right)\right) \stackrel{(E 1)}{=}\left(\top,\left(a^{\dagger} \cap c^{\dagger} \cap b \cap a\right) \cup\left(a^{\dagger} \cap c^{\dagger} \cap d \cap c\right)\right)=(\top, \perp)=1$. Besides we have that $\left((a, b)^{\prime} \wedge(c, d)^{\prime}\right) \rightarrow((a, b) \vee(c, d))^{\prime}=\left(\left(a^{\dagger}, b \cap\left(a^{\dagger} \Rightarrow a\right)\right) \wedge\left(c^{\dagger}, d \cap\left(c^{\dagger} \Rightarrow c\right)\right)\right) \rightarrow$ $(a \cup c, b \cap d)^{\prime}=\left(a^{\dagger} \cap c^{\dagger},\left(b \cap\left(a^{\dagger} \Rightarrow a\right)\right) \cup\left(d \cap\left(c^{\dagger} \Rightarrow c\right)\right)\right) \rightarrow\left((a \cup c)^{\dagger},(b \cap d) \cap\left((a \cup c)^{\dagger} \Rightarrow\right.\right.$ $(a \cup c))) \stackrel{(E 8)}{=}\left(a^{\dagger} \cap c^{\dagger},\left(b \cap\left(a^{\dagger} \Rightarrow a\right)\right) \cup\left(d \cap\left(c^{\dagger} \Rightarrow c\right)\right)\right) \rightarrow\left(\left(a^{\dagger} \cap c^{\dagger}\right),(b \cap d) \cap\left(\left(a^{\dagger} \cap c^{\dagger}\right) \Rightarrow\right.\right.$ $(a \cup c)))=\left(\left(a^{\dagger} \cap c^{\dagger}\right) \Rightarrow\left(a^{\dagger} \cap c^{\dagger}\right),\left(a^{\dagger} \cap c^{\dagger}\right) \cap(b \cap d) \cap\left(\left(a^{\dagger} \cap c^{\dagger}\right) \Rightarrow(a \cup c)\right)\right) \stackrel{(E 3)}{=}$ $\left(\mathrm{T},\left(a^{\dagger} \cap c^{\dagger}\right) \cap(b \cap d) \cap\left(\left(a^{\dagger} \cap c^{\dagger}\right) \Rightarrow(a \cup c)\right)\right) \stackrel{(E 1)}{=}\left(\top, a^{\dagger} \cap c^{\dagger} \cap b \cap d \cap(a \cup c)\right)=(\top, \perp)=1$.

3. $\mathbb{A}=\mathbb{D} m s \mathbb{S H}$ : as in the Case 2 we have that $\mathbf{V}_{k}(\mathbf{A}) \in \mathbb{O C K \mathbb { S N }}$. Then we only need compute the following calculus: $(a, b)^{\prime \prime} \rightarrow\left((a, b)^{\prime \prime} \wedge(a, b)\right) \stackrel{(4.10)}{=}\left(a^{\dagger \dagger}, b \cap\left(a^{\dagger} \Rightarrow a\right) \cap\left(a^{\dagger \dagger} \Rightarrow a^{\dagger}\right)\right) \rightarrow$ $\left(\left(a^{\dagger \dagger}, b \cap\left(a^{\dagger} \Rightarrow a\right) \cap\left(a^{\dagger \dagger} \Rightarrow a^{\dagger}\right)\right) \wedge(a, b)\right)=\left(a^{\dagger \dagger}, b \cap\left(a^{\dagger} \Rightarrow a\right) \cap\left(a^{\dagger \dagger} \Rightarrow a^{\dagger}\right)\right) \rightarrow$ $\left(a^{\dagger \dagger} \cap a, b\right) \stackrel{(E 9)}{=}\left(a^{\dagger \dagger}, b \cap\left(a^{\dagger} \Rightarrow a\right) \cap\left(a^{\dagger \dagger} \Rightarrow a^{\dagger}\right)\right) \rightarrow\left(a^{\dagger \dagger}, b\right)=\left(a^{\dagger \dagger} \Rightarrow a^{\dagger \dagger}, a^{\dagger \dagger} \cap b\right) \stackrel{(E 9)}{=}$ $\left(\top, a^{\dagger \dagger} \cap a \cap b\right)=(\top, \perp)=1$.

4. $\mathbb{A}=\mathbb{D M S H}$ : as in the Case $2, \mathbf{V}_{k}(\mathbf{A}) \in \mathbb{O} \mathbb{C} \mathbb{K S N}$. Taking into account a similar computation to that of the Case 3 we obtain that $(a, b)^{\prime \prime} \rightarrow\left((a, b)^{\prime \prime} \wedge(a, b)\right)=1$. Note that $(a, b) \rightarrow$ $\left((a, b) \wedge(a, b)^{\prime \prime}\right) \stackrel{(4.10)}{=}(a, b) \rightarrow\left((a, b) \wedge\left(a^{\dagger \dagger}, b \cap\left(a^{\dagger} \Rightarrow a\right) \cap\left(a^{\dagger \dagger} \Rightarrow a^{\dagger}\right)\right)\right)=(a, b) \rightarrow$ $\left(a \cap a^{\dagger \dagger}, b\right) \stackrel{(E 10)}{=}(a, b) \rightarrow(a, b)=1$.

5. $\mathbb{A}=\mathbb{D S D S H}$ : taking into account (4.10) we have that

$$
\begin{aligned}
& (a, b)^{\prime \prime \prime} \stackrel{(4.10)}{=}\left(a^{\dagger \dagger}, b \cap\left(a^{\dagger} \Rightarrow a\right) \cap\left(a^{\dagger \dagger} \Rightarrow a^{\dagger}\right)\right)^{\prime} \\
& =\left(a^{\dagger \dagger}, b \cap\left(a^{\dagger} \Rightarrow a\right) \cap\left(a^{\dagger \dagger} \Rightarrow a^{\dagger}\right) \cap\left(a^{\dagger \dagger \dagger} \Rightarrow a^{\dagger \dagger}\right)\right) \text {, } \\
& (a, b)^{\prime \prime} \vee(c, d)^{\prime \prime}=\left(a^{\dagger \dagger} \cup c^{\dagger \dagger}, b \cap\left(a^{\dagger} \Rightarrow a\right) \cap\left(a^{\dagger \dagger} \Rightarrow a^{\dagger}\right) \cap d \cap\left(c^{\dagger} \Rightarrow c\right) \cap\left(c^{\dagger \dagger} \Rightarrow c^{\dagger}\right)\right)
\end{aligned}
$$

and

$$
((a, b) \vee(c, d))^{\prime \prime}=\left((a \cup c)^{\dagger \dagger},(b \cap d) \cap\left((a \cup c)^{\dagger} \Rightarrow(a \cup c)\right) \cap\left((a \cup c)^{\dagger \dagger} \Rightarrow(a \cup c)^{\dagger}\right)\right)
$$

Now we will prove the equation $(E 40):((a, b) \vee(c, d))^{\prime \prime} \rightarrow\left((a, b)^{\prime \prime} \vee(c, d)^{\prime \prime}\right) \stackrel{(4.12)}{=}(a \cup c$, $b \cap d)^{\prime \prime} \rightarrow\left(a^{\dagger \dagger} \cup c^{\dagger \dagger}, b \cap\left(a^{\dagger} \Rightarrow a\right) \cap\left(a^{\dagger \dagger} \Rightarrow a^{\dagger}\right) \cap d \cap\left(c^{\dagger} \Rightarrow c\right) \cap\left(c^{\dagger \dagger} \Rightarrow c^{\dagger}\right)\right) \stackrel{(4.13)}{=}$ $\left((a \cup c)^{\dagger \dagger},(b \cap d) \cap\left((a \cup c)^{\dagger} \Rightarrow(a \cup c)\right) \cap\left((a \cup c)^{\dagger \dagger} \Rightarrow(a \cup c)^{\dagger}\right)\right) \rightarrow\left(a^{\dagger \dagger} \cup c^{\dagger \dagger}, b \cap\right.$ $\left.\left(a^{\dagger} \Rightarrow a\right) \cap\left(a^{\dagger \dagger} \Rightarrow a^{\dagger}\right) \cap d \cap\left(c^{\dagger} \Rightarrow c\right) \cap\left(c^{\dagger \dagger} \Rightarrow c^{\dagger}\right)\right)=\left((a \cup c)^{\dagger \dagger} \Rightarrow\left(a^{\dagger \dagger} \cup c^{\dagger \dagger}\right),(a \cup c)^{\dagger \dagger} \cap\right.$ $\left.b \cap\left(a^{\dagger} \Rightarrow a\right) \cap\left(a^{\dagger \dagger} \Rightarrow a^{\dagger}\right) \cap d \cap\left(c^{\dagger} \Rightarrow c\right) \cap\left(c^{\dagger \dagger} \Rightarrow c^{\dagger}\right)\right) \stackrel{(E 11)}{=}\left(\left(a^{\dagger \dagger} \cup c^{\dagger \dagger}\right) \Rightarrow\left(a^{\dagger \dagger} \cup c^{\dagger \dagger}\right),\left(a^{\dagger \dagger} \cup\right.\right.$ $\left.\left.c^{\dagger \dagger}\right) \cap b \cap\left(a^{\dagger} \Rightarrow a\right) \cap\left(a^{\dagger \dagger} \Rightarrow a^{\dagger}\right) \cap d \cap\left(c^{\dagger} \Rightarrow c\right) \cap\left(c^{\dagger \dagger} \Rightarrow c^{\dagger}\right)\right)=\left(\top,\left(a^{\dagger \dagger} \cup c^{\dagger \dagger}\right) \cap b \cap\left(a^{\dagger} \Rightarrow\right.\right.$ $\left.a) \cap\left(a^{\dagger \dagger} \Rightarrow a^{\dagger}\right) \cap d \cap\left(c^{\dagger} \Rightarrow c\right) \cap\left(c^{\dagger \dagger} \Rightarrow c^{\dagger}\right)\right) \stackrel{(E 1)}{=}(\top, \perp)=1$. The equation (E41) is proved as follows: $=\left((a, b)^{\prime \prime} \vee(c, d)^{\prime \prime}\right) \rightarrow((a, b) \vee(c, d))^{\prime \prime} \stackrel{(4.13)}{=}\left(a^{\dagger \dagger} \cup c^{\dagger \dagger}, b \cap\left(a^{\dagger} \Rightarrow a\right) \cap\left(a^{\dagger \dagger} \Rightarrow\right.\right.$ $\left.\left.a^{\dagger}\right) \cap d \cap\left(c^{\dagger} \Rightarrow c\right) \cap\left(c^{\dagger \dagger} \Rightarrow c^{\dagger}\right)\right) \rightarrow((a, b) \vee(c, d))^{\prime \prime} \stackrel{(4.12)}{=}\left(a^{\dagger \dagger} \cup c^{\dagger \dagger}, b \cap\left(a^{\dagger} \Rightarrow a\right) \cap\left(a^{\dagger \dagger} \Rightarrow\right.\right.$ $\left.\left.a^{\dagger}\right) \cap d \cap\left(c^{\dagger} \Rightarrow c\right) \cap\left(c^{\dagger \dagger} \Rightarrow c^{\dagger}\right)\right) \rightarrow\left((a \cup c)^{\dagger \dagger},(b \cap d) \cap\left((a \cup c)^{\dagger} \Rightarrow(a \cup c)\right) \cap\left((a \cup c)^{\dagger \dagger} \Rightarrow\right.\right.$ $\left.\left.(a \cup c)^{\dagger}\right)\right)=\left(\left(a^{\dagger \dagger} \cup c^{\dagger \dagger}\right) \Rightarrow(a \cup c)^{\dagger \dagger},\left(a^{\dagger \dagger} \cup c^{\dagger \dagger}\right) \cap(b \cap d) \cap\left((a \cup c)^{\dagger} \Rightarrow(a \cup c)\right) \cap\left((a \cup c)^{\dagger \dagger} \Rightarrow\right.\right.$ $\left.\left.(a \cup c)^{\dagger}\right)\right) \stackrel{(E 11)}{=}\left(\top,\left(a^{\dagger \dagger} \cup c^{\dagger \dagger}\right) \cap(b \cap d) \cap\left((a \cup c)^{\dagger} \Rightarrow(a \cup c)\right) \cap\left(\left(a^{\dagger \dagger} \cup c^{\dagger \dagger}\right) \Rightarrow(a \cup c)^{\dagger}\right)\right) \stackrel{(E 1)}{=}$ 
$\left(\top,\left(a^{\dagger \dagger} \cup c^{\dagger \dagger}\right) \cap(b \cap d) \cap\left((a \cup c)^{\dagger} \Rightarrow(a \cup c)\right) \cap(a \cup c)^{\dagger}\right) \stackrel{(E 1)}{=}\left(\top,\left(a^{\dagger \dagger} \cup c^{\dagger \dagger}\right) \cap b(b \cap\right.$ d) $\left.\cap(a \cup c) \cap(a \cup c)^{\dagger}\right) \stackrel{(E 1)}{=}(\top, \perp)=1$. The equation $(E 42)$ can be proved as follows: $(a, b)^{\prime \prime \prime} \rightarrow(a, b)^{\prime} \stackrel{(4.12)}{=}\left(a^{\dagger \dagger \dagger}, b \cap\left(a^{\dagger} \Rightarrow a\right) \cap\left(a^{\dagger \dagger} \Rightarrow a^{\dagger}\right) \cap\left(a^{\dagger \dagger \dagger} \Rightarrow a^{\dagger \dagger}\right)\right) \rightarrow(a, b)^{\prime}=$ $\left(a^{\dagger \dagger}, b \cap\left(a^{\dagger} \Rightarrow a\right) \cap\left(a^{\dagger \dagger} \Rightarrow a^{\dagger}\right) \cap\left(a^{\dagger \dagger \dagger} \Rightarrow a^{\dagger \dagger}\right)\right) \rightarrow\left(a^{\dagger}, b \cap\left(a^{\dagger} \Rightarrow a\right)\right) \stackrel{(E 12)}{=}\left(a^{\dagger}, b \cap\right.$ $\left.\left(a^{\dagger} \Rightarrow a\right) \cap\left(a^{\dagger \dagger} \Rightarrow a^{\dagger}\right) \cap\left(a^{\dagger \dagger \dagger} \Rightarrow a^{\dagger \dagger}\right)\right) \rightarrow\left(a^{\dagger}, b \cap\left(a^{\dagger} \Rightarrow a\right)\right)=\left(a^{\dagger} \Rightarrow a^{\dagger}, a^{\dagger} \cap b \cap\right.$ $\left.\left(a^{\dagger} \Rightarrow a\right)\right)=\left(\top, a^{\dagger} \cap b \cap\left(a^{\dagger} \Rightarrow a\right)\right) \stackrel{(E 1)}{=}(\top, \perp)=1$. Finally, the equation $(E 43)$ is satisfied because $(a, b)^{\prime} \rightarrow(a, b)^{\prime \prime \prime} \stackrel{(4.11)}{=}(a, b)^{\prime} \rightarrow\left(a^{\dagger \dagger \dagger}, b \cap\left(a^{\dagger} \Rightarrow a\right) \cap\left(a^{\dagger \dagger} \Rightarrow a^{\dagger}\right) \cap\left(a^{\dagger \dagger \dagger} \Rightarrow a^{\dagger \dagger}\right)\right)=$ $\left(a^{\dagger}, b \cap\left(a^{\dagger} \Rightarrow a\right)\right) \rightarrow\left(a^{\dagger \dagger \dagger}, b \cap\left(a^{\dagger} \Rightarrow a\right) \cap\left(a^{\dagger \dagger} \Rightarrow a^{\dagger}\right) \cap\left(a^{\dagger \dagger \dagger} \Rightarrow a^{\dagger \dagger}\right)\right) \stackrel{(E 12)}{=}\left(a^{\dagger}, b \cap\left(a^{\dagger} \Rightarrow\right.\right.$ a) $) \rightarrow\left(a^{\dagger}, b \cap\left(a^{\dagger} \Rightarrow a\right) \cap\left(a^{\dagger \dagger} \Rightarrow a^{\dagger}\right) \cap\left(a^{\dagger \dagger \dagger} \Rightarrow a^{\dagger \dagger}\right)\right)=\left(a^{\dagger} \Rightarrow a^{\dagger}, a^{\dagger} \cap b \cap\left(a^{\dagger} \Rightarrow\right.\right.$ a) $\left.\cap\left(a^{\dagger \dagger} \Rightarrow a^{\dagger}\right) \cap\left(a^{\dagger \dagger} \Rightarrow a^{\dagger \dagger}\right)\right) \stackrel{(E 1)}{=}\left(a^{\dagger} \Rightarrow a^{\dagger}, \perp\right)=(\top, \perp)=1$.

6. $\mathbb{A}=\mathbb{D Q D D S H}$ : as in the Case 5 we have that $\mathbf{V}_{k}(\mathbf{A}) \in \mathbb{D S D S N}$. Then it can be proved $(a, b)^{\prime \prime} \rightarrow$ $\left((a, b)^{\prime \prime} \wedge(a, b)\right)=1$ as in the Case 3 .

7. $\mathbb{A}=\mathbb{D P C S H}$ : as in the Case $6, \mathbf{V}_{k}(\mathbf{A}) \in \mathbb{D Q D D N}$. Finally, we need to show the following: $(a, b) \vee(a, b)^{\prime}=(a, b) \vee\left(a^{\dagger}, b \cap\left(a^{\dagger} \Rightarrow a\right)\right)=\left(a \cup a^{\dagger}, a \cap b \cap\left(a^{\dagger} \Rightarrow a\right)\right)=\left(a \cup a^{\dagger}, \perp\right) \stackrel{(E 13)}{=}$ $(\top, \perp)=1$.

8. $\mathbb{A}=\mathbb{D Q Q B B S H}$ : as in the Case $6, \mathbf{V}_{k}(\mathbf{A}) \in \mathbb{D} \mathbb{Q D S N}$. Besides, $(a, b) \vee((a, b) \rightarrow(\sim 1))=$ $(a, b) \vee((a, b) \rightarrow(\sim(\top, \perp)))=(a, b) \vee((a, b) \rightarrow(\perp, \top))=(a, b) \vee(a \Rightarrow \perp, a)=(a \cup(a \Rightarrow$ $\perp), \perp) \stackrel{(E 15)}{=}(\top, \perp)=1$.

9. $\mathbb{A}=\mathbb{D Q S S H}:$ we have that $(a, b)^{\prime \prime} \rightarrow\left((a, b)^{\prime \prime} \wedge(a, b)\right)=1$ as in the Case 3. Note that in this case $(a, b)^{\prime} \wedge(c, d)^{\prime \prime}=\left(a^{\dagger}, b \cap\left(a^{\dagger} \Rightarrow a\right)\right) \wedge(c, d)^{\prime \prime} \stackrel{(4.10)}{=}\left(a^{\dagger}, b \cap\left(a^{\dagger} \Rightarrow a\right)\right) \wedge\left(c^{\dagger \dagger}, d \cap\right.$ $\left.\left(c^{\dagger} \Rightarrow c\right) \cap\left(c^{\dagger \dagger} \Rightarrow c^{\dagger}\right)\right)=\left(a^{\dagger} \cap c^{\dagger \dagger},\left(b \cap\left(a^{\dagger} \Rightarrow a\right)\right) \cup\left(d \cap\left(c^{\dagger} \Rightarrow c\right) \cap\left(c^{\dagger \dagger} \Rightarrow c^{\dagger}\right)\right)\right)$. Thus,

$$
(a, b)^{\prime} \wedge(c, d)^{\prime \prime}=\left(a^{\dagger} \cap c^{\dagger \dagger},\left(b \cap\left(a^{\dagger} \Rightarrow a\right)\right) \cup\left(d \cap\left(c^{\dagger} \Rightarrow c\right) \cap\left(c^{\dagger \dagger} \Rightarrow c^{\dagger}\right)\right)\right) .
$$

Besides, $\left((a, b) \vee(c, d)^{\prime}\right)^{\prime}=\left((a, b) \vee\left(c^{\dagger}, d \cap\left(c^{\dagger} \Rightarrow c\right)\right)\right)^{\prime}=\left(a \cup c^{\dagger}, b \cap d \cap\left(c^{\dagger} \Rightarrow\right.\right.$ $c))^{\prime}=\left(\left(a \cup c^{\dagger}\right)^{\dagger}, b \cap d \cap\left(c^{\dagger} \Rightarrow c\right) \cap\left(\left(a \cup c^{\dagger}\right)^{\dagger} \Rightarrow\left(a \cup c^{\dagger}\right)\right)\right) \stackrel{(E 16)}{=}\left(a^{\dagger} \cap c^{\dagger \dagger}, b \cap d \cap\right.$ $\left.\left(c^{\dagger} \Rightarrow c\right) \cap\left(\left(a^{\dagger} \cap c^{\dagger \dagger}\right) \Rightarrow\left(a \cup c^{\dagger}\right)\right)\right)$. Thus,

$$
\left((a, b) \vee(c, d)^{\prime}\right)^{\prime}=\left(a^{\dagger} \cap c^{\dagger \dagger}, b \cap d \cap\left(c^{\dagger} \Rightarrow c\right) \cap\left(\left(a^{\dagger} \cap c^{\dagger \dagger}\right) \Rightarrow\left(a \cup c^{\dagger}\right)\right)\right) .
$$

Now we will prove the equations $(E 45),(E 46)$ and (E47).

- $(E 45):\left((a, b)^{\prime} \wedge(a, b)^{\prime \prime}\right) \rightarrow(\sim 1) \stackrel{(4.10)}{=}\left((a, b)^{\prime} \wedge\left(a^{\dagger \dagger}, b \cap\left(a^{\dagger} \Rightarrow a\right) \cap\left(a^{\dagger \dagger} \Rightarrow a^{\dagger}\right)\right)\right) \rightarrow$ $(\sim 1)=\left(\left(a^{\dagger}, b \cap\left(a^{\dagger} \Rightarrow a\right)\right) \wedge\left(a^{\dagger \dagger}, b \cap\left(a^{\dagger} \Rightarrow a\right) \cap\left(a^{\dagger \dagger} \Rightarrow a^{\dagger}\right)\right)\right) \rightarrow(\sim 1)=$ $\left(a^{\dagger} \cap a^{\dagger \dagger}, b \cap\left(a^{\dagger} \Rightarrow a\right)\right) \rightarrow(\sim 1) \stackrel{(E 17)}{=}\left(\perp, b \cap\left(a^{\dagger} \Rightarrow a\right)\right) \rightarrow(\sim 1)=(\perp, b \cap$ $\left.\left(a^{\dagger} \Rightarrow a\right)\right) \rightarrow(\perp, \top)=(\top, \perp)=1$.

- $(E 46):\left((a, b)^{\prime} \wedge(c, d)^{\prime \prime}\right) \rightarrow\left(\left((a, b) \vee(c, d)^{\prime}\right)^{\prime}\right) \stackrel{(4.14)}{=}\left(a^{\dagger} \cap c^{\dagger \dagger},\left(b \cap\left(a^{\dagger} \Rightarrow a\right)\right) \cup(d \cap\right.$ $\left.\left.\left(c^{\dagger} \Rightarrow c\right) \cap\left(c^{\dagger \dagger} \Rightarrow c^{\dagger}\right)\right)\right) \rightarrow\left(\left((a, b) \vee(c, d)^{\prime}\right)^{\prime}\right) \stackrel{(4.15)}{=}\left(a^{\dagger} \cap c^{\dagger \dagger},\left(b \cap\left(a^{\dagger} \Rightarrow a\right)\right) \cup(d \cap\right.$ $\left.\left.\left(c^{\dagger} \Rightarrow c\right) \cap\left(c^{\dagger \dagger} \Rightarrow c^{\dagger}\right)\right)\right) \rightarrow\left(a^{\dagger} \cap c^{\dagger \dagger}, b \cap d \cap\left(c^{\dagger} \Rightarrow c\right) \cap\left(\left(a^{\dagger} \cap c^{\dagger \dagger}\right) \Rightarrow\left(a \cup c^{\dagger}\right)\right)\right)$. $=\left(\top, a^{\dagger} \cap c^{\dagger \dagger} \cap b \cap d \cap\left(c^{\dagger} \Rightarrow c\right) \cap\left(\left(a^{\dagger} \cap c^{\dagger \dagger}\right) \Rightarrow\left(a \cup c^{\dagger}\right)\right)\right) \stackrel{(E 1)}{=}\left(\top, a^{\dagger} \cap c^{\dagger \dagger} \cap b \cap d \cap\right.$ $\left.\left(c^{\dagger} \Rightarrow c\right) \cap\left(a \cup c^{\dagger}\right)\right)=\left(\top,\left(a^{\dagger} \cap c^{\dagger \dagger} \cap b \cap d \cap\left(c^{\dagger} \Rightarrow c\right) \cap a\right) \cup\left(a^{\dagger} \cap c^{\dagger \dagger} \cap b \cap d \cap\right.\right.$ $\left.\left.\left(c^{\dagger} \Rightarrow c\right) \cap c^{\dagger}\right)\right)=\left(\top, \perp \cup\left(a^{\dagger} \cap c^{\dagger \dagger} \cap b \cap d \cap\left(c^{\dagger} \Rightarrow c\right) \cap c^{\dagger}\right)\right) \stackrel{(E 1)}{=}\left(\top, \perp \cup\left(a^{\dagger} \cap c^{\dagger \dagger} \cap\right.\right.$ $\left.\left.b \cap d \cap c \cap c^{\dagger}\right)\right)=(\top, \perp)=1$. 
- $(E 47):=\left(\left((a, b) \vee(c, d)^{\prime}\right)^{\prime}\right) \rightarrow\left((a, b)^{\prime} \wedge(c, d)^{\prime \prime}\right) \stackrel{(4.14)}{=}\left(\left((a, b) \vee(c, d)^{\prime}\right)^{\prime}\right) \rightarrow$ $\left(a^{\dagger} \cap c^{\dagger \dagger},\left(b \cap\left(a^{\dagger} \Rightarrow a\right)\right) \cup\left(d \cap\left(c^{\dagger} \Rightarrow c\right) \cap\left(c^{\dagger \dagger} \Rightarrow c^{\dagger}\right)\right)\right) \stackrel{(4.15)}{=}\left(a^{\dagger} \cap c^{\dagger \dagger}, b \cap d \cap\right.$ $\left.\left(c^{\dagger} \Rightarrow c\right) \cap\left(\left(a^{\dagger} \cap c^{\dagger \dagger}\right) \Rightarrow\left(a \cup c^{\dagger}\right)\right)\right) \rightarrow\left(a^{\dagger} \cap c^{\dagger \dagger},\left(b \cap\left(a^{\dagger} \Rightarrow a\right)\right) \cup\left(d \cap\left(c^{\dagger} \Rightarrow c\right) \cap\left(c^{\dagger \dagger} \Rightarrow\right.\right.\right.$ $\left.\left.\left.c^{\dagger}\right)\right)\right)=\left(\top, a^{\dagger} \cap c^{\dagger \dagger} \cap\left(\left(b \cap\left(a^{\dagger} \Rightarrow a\right)\right) \cup\left(d \cap\left(c^{\dagger} \Rightarrow c\right) \cap\left(c^{\dagger \dagger} \Rightarrow c^{\dagger}\right)\right)\right)\right)=\left(\top,\left(a^{\dagger} \cap c^{\dagger \dagger} \cap\right.\right.$ $\left.\left.b \cap\left(a^{\dagger} \Rightarrow a\right)\right) \cup\left(a^{\dagger} \cap c^{\dagger \dagger} \cap d \cap\left(c^{\dagger} \Rightarrow c\right) \cap\left(c^{\dagger \dagger} \Rightarrow c^{\dagger}\right)\right)\right) \stackrel{(E 1)}{=}\left(\top,\left(a^{\dagger} \cap c^{\dagger \dagger} \cap b \cap a\right) \cup\left(a^{\dagger} \cap\right.\right.$ $\left.\left.c^{\dagger \dagger} \cap d \cap\left(c^{\dagger} \Rightarrow c\right) \cap c^{\dagger}\right)\right) \stackrel{(E 1)}{=}\left(\top,\left(a^{\dagger} \cap c^{\dagger \dagger} \cap b \cap a\right) \cup\left(a^{\dagger} \cap c^{\dagger \dagger} \cap d \cap c \cap c^{\dagger}\right)\right)=(\top, \perp)=1$.

10. $\mathbb{A}=\mathbb{D S S H}$ : the equations (E36) and (E37) can be proved as in the Case 2 . The equation (E38) can be showed as in the Case 3. Finally, the equations (E45), (E46) and (E47) can be checked as in the Case 9.

11. $\mathbb{A}=\mathbb{B D Q S S H H}:$ in this case note that $((a, b) \vee((a, b) \rightarrow(\sim(\top, \perp))))^{\prime}=((a, b) \vee((a, b) \rightarrow$ $(\perp, \top)))^{\prime}=((a, b) \vee(a \Rightarrow \perp, a))^{\prime}=(a \cup(a \Rightarrow \perp), \perp)^{\prime}=\left((a \cup(a \Rightarrow \perp))^{\dagger}, \perp\right)=((a \cup$ $\left.(a \Rightarrow \perp))^{\dagger}, \perp\right) \stackrel{(E 14)}{=}\left(a^{\dagger} \cap(a \Rightarrow \perp)^{\dagger}, \perp\right)$ and $(a, b)^{\prime} \wedge((a, b) \rightarrow(\sim(\top, \perp)))^{\prime}=(a, b)^{\prime} \wedge$ $((a, b) \rightarrow(\perp, \top))^{\prime}=(a, b)^{\prime} \wedge(a \Rightarrow \perp, a)^{\prime}=(a, b)^{\prime} \wedge(a \Rightarrow \perp, a)^{\prime}=\left(a^{\dagger}, b \cap\left(a^{\dagger} \Rightarrow a\right)\right) \wedge$ $\left((a \Rightarrow \perp)^{\dagger}, a \cap\left((a \Rightarrow \perp)^{\dagger} \Rightarrow(a \Rightarrow \perp)\right)\right)=\left(a^{\dagger} \cap(a \Rightarrow \perp)^{\dagger},\left(b \cap\left(a^{\dagger} \Rightarrow a\right)\right) \cup(a \cap\right.$ $\left.\left.\left((a \Rightarrow \perp)^{\dagger} \Rightarrow(a \Rightarrow \perp)\right)\right)\right) \stackrel{(E 14)}{=}\left(a^{\dagger} \cap(a \Rightarrow \perp)^{\dagger},\left(b \cap\left(a^{\dagger} \Rightarrow a\right)\right) \cup\left(a \cap\left((a \Rightarrow \perp)^{\dagger} \Rightarrow(a \Rightarrow\right.\right.\right.$ $\perp)))$. Thus,

$$
((a, b) \vee((a, b) \rightarrow(\sim(\top, \perp))))^{\prime}=\left(a^{\dagger} \cap(a \Rightarrow \perp)^{\dagger}, \perp\right)
$$

and

$$
\begin{gathered}
(a, b)^{\prime} \wedge((a, b) \rightarrow(\sim(\top, \perp)))^{\prime}=\left(a^{\dagger} \cap(a \Rightarrow \perp)^{\dagger},\right. \\
\left.\left(b \cap\left(a^{\dagger} \Rightarrow a\right)\right) \cup\left(a \cap\left((a \Rightarrow \perp)^{\dagger} \Rightarrow(a \Rightarrow \perp)\right)\right)\right) .
\end{gathered}
$$

Hence, $((a, b) \vee((a, b) \rightarrow(\sim(\top, \perp))))^{\prime} \rightarrow\left((a, b)^{\prime} \wedge((a, b) \rightarrow(\sim(\top, \perp)))^{\prime}\right) \stackrel{(4.16) \text { and (4.17) }}{=}$ $\left(a^{\dagger} \cap(a \Rightarrow \perp)^{\dagger}, \perp\right) \rightarrow\left(a^{\dagger} \cap(a \Rightarrow \perp)^{\dagger},\left(b \cap\left(a^{\dagger} \Rightarrow a\right)\right) \cup\left(a \cap\left((a \Rightarrow \perp)^{\dagger} \Rightarrow(a \Rightarrow \perp)\right)\right)\right)=$ $\left(\top,\left(a^{\dagger} \cap(a \Rightarrow \perp)^{\dagger}\right) \cap\left(\left(b \cap\left(a^{\dagger} \Rightarrow a\right)\right) \cup\left(a \cap\left((a \Rightarrow \perp)^{\dagger} \Rightarrow(a \Rightarrow \perp)\right)\right)\right)=\left(\top,\left(a^{\dagger} \cap(a \Rightarrow\right.\right.\right.$ $\left.\perp)^{\dagger} \cap b \cap\left(a^{\dagger} \Rightarrow a\right)\right) \cup\left(a^{\dagger} \cap(a \Rightarrow \perp)^{\dagger} \cap a \cap\left((a \Rightarrow \perp)^{\dagger} \Rightarrow(a \Rightarrow \perp)\right)\right) \stackrel{(E 1)}{=}\left(\top,\left(a^{\dagger} \cap\right.\right.$ $\left.(a \Rightarrow \perp)^{\dagger} \cap b \cap a\right) \cup\left(a^{\dagger} \cap(a \Rightarrow \perp)^{\dagger} \cap a \cap(a \Rightarrow \perp)\right) \stackrel{(E 1)}{=}\left(\top,\left(a^{\dagger} \cap(a \Rightarrow \perp)^{\dagger} \cap b \cap a\right) \cup\left(a^{\dagger} \cap\right.\right.$ $\left.\left.(a \Rightarrow \perp)^{\dagger} \cap a \cap \perp\right)\right)=(\top, \perp)=1$. Besides, $\left((a, b)^{\prime} \wedge((a, b) \rightarrow(\sim(\top, \perp)))^{\prime}\right) \rightarrow((a, b) \vee$ $((a, b) \rightarrow(\sim(\top, \perp))))^{\prime} \stackrel{(4.16) \text { and }{ }^{(4.17)}}{=}\left(a^{\dagger} \cap(a \Rightarrow \perp)^{\dagger},\left(b \cap\left(a^{\dagger} \Rightarrow a\right)\right) \cup\left(a \cap\left((a \Rightarrow \perp)^{\dagger} \Rightarrow\right.\right.\right.$ $(a \Rightarrow \perp)))) \rightarrow\left(a^{\dagger} \cap(a \Rightarrow \perp)^{\dagger}, \perp\right)=(\top, \perp)=1$. Then $\mathbf{V}_{k}(\mathbf{A})$ satisfies the equations $(E 48)$ and (E49). The rest of the equations can be verified as in the Case 9.

12. $\mathbb{A}=\mathbb{D S C S N}$ : the proof is similar to that of the Case 7 .

13. $\mathbb{A} \in\{\mathbb{D M H}, \mathbb{D P C H}\}$ : The fact that $\mathbf{A}$ is in $\mathbb{D M S H}$ and in $\mathbb{D P C S H}$ can be proved as in the Cases 4 and 7, respectively. Then the proof can be finished following the Case 1 .

\subsection{Representations}

In this section we show that there exists a representation of dually hemimorphic semi-Nelson algebras as subalgebras of the Vakarelov construction applied to a suitable dually hemimorphic semi-Heyting algebra, much in the same manner as in the case of semi-Nelson (Nelson) and semiHeyting (Heyting) algebras. In the other direction, we can also represent every dually hemimorphic semi-Heyting algebra as a quotient of a dually hemimorphic semi-Nelson one.

Let $\mathbf{A}=(A, \wedge, \vee, \rightarrow, \sim, 1) \in \mathbb{S N}$. It follows from the proof of [10, Corollary 5.2] that the map $h: \mathbf{A} \rightarrow\left(\mathbf{V}_{k}(\mathbf{s H}(\mathbf{A}))\right)$ defined by $h(a)=(\llbracket a \rrbracket, \llbracket \sim a \rrbracket)$ is an injective morphism. 
THEOREM 4.8

If $\mathbf{A}=\left(A, \wedge, \vee, \rightarrow, \sim,{ }^{\prime}, 1\right) \in \mathbb{D H M} \mathbb{S N}$ then $\mathbf{A}$ is isomorphic to a subalgebra of $\mathbf{V}_{k}(\mathbf{s H}(\mathbf{A}))$.

ProOF. We only need to check that the map $h$ preserves the operation '. Observe that $h\left(a^{\prime}\right)=$ $\left(\llbracket a^{\prime} \rrbracket, \llbracket \sim a^{\prime} \rrbracket\right)$ and

$$
(h(a))^{\prime}=(\llbracket a \rrbracket, \llbracket \sim a \rrbracket)^{\prime}=\left(\llbracket a \rrbracket^{\dagger}, \llbracket \sim a \rrbracket \cap\left(\llbracket a \rrbracket^{\dagger} \Rightarrow \llbracket a \rrbracket\right)\right)=\left(\llbracket a^{\prime} \rrbracket, \llbracket \sim a \cap\left(a^{\prime} \rightarrow a\right) \rrbracket\right) .
$$

Since A satisfies the equalities (E32) and (E33) then $\llbracket \sim a^{\prime} \rrbracket=\llbracket \sim a \cap\left(a^{\prime} \rightarrow a\right) \rrbracket$. Hence, $h\left(a^{\prime}\right)=$ $(h(a))^{\prime}$.

In semi-Heyting algebras we can define the pseudocomplement of an element $a$ as $a^{*}=a \Rightarrow 0$. In particular, $a^{*}=a \Rightarrow_{N} 0$ (see [21]). Let $\mathbf{A}=(A, \cap, \cup, \Rightarrow, \perp, \top) \in \mathbb{S H}$. The map $i: \mathbf{A} \rightarrow \mathbf{s H}\left(\mathbf{V}_{k}(\mathbf{A})\right)$ defined as $i(a)=\llbracket\left(a, a^{*}\right) \rrbracket$ is an isomorphism, as it was showed in the proof of [10, Theorem 5.3].

THEOREM 4.9

If $\mathbf{A}=\left(A, \cap, \cup, \Rightarrow,{ }^{\dagger}, \perp, \top\right)$ is a dually hemimorphic semi-Heyting algebra then $\mathbf{A}$ is isomorphic to $\mathbf{s H}\left(\mathbf{V}_{k}(\mathbf{A})\right)$.

ProOF. We only need to show that the map $i$ preserves the unary operation ${ }^{\dagger}$. Note that

$$
i\left(a^{\dagger}\right)=\llbracket\left(a^{\dagger},\left(a^{\dagger}\right)^{*}\right) \rrbracket .
$$

We also have that

$$
(i(a))^{\dagger}=\llbracket\left(a, a^{*}\right) \rrbracket^{\dagger}=\llbracket\left(a, a^{*}\right)^{\prime} \rrbracket=\llbracket\left(a^{\dagger}, a^{*} \cap\left(a^{\dagger} \Rightarrow a\right)\right) \rrbracket .
$$

Since

$$
\left(a^{\dagger}, a^{*} \cap\left(a^{\dagger} \Rightarrow a\right)\right) \rightarrow\left(a^{\dagger},\left(a^{\dagger}\right)^{*}\right)=\left(\top, a^{\dagger} \cap\left(a^{\dagger}\right)^{*}\right)=(\top, \perp)=1
$$

and

$$
\left(a^{\dagger},\left(a^{\dagger}\right)^{*}\right) \rightarrow\left(a^{\dagger}, a^{*} \cap\left(a^{\dagger} \Rightarrow a\right)\right)=\left(\top, a^{\dagger} \cap a^{*} \cap\left(a^{\dagger} \Rightarrow a\right)\right)=\left(\top, a^{\dagger} \cap a^{*} \cap a\right)=(\top, \perp)=1
$$

we have that $\llbracket\left(a^{\dagger},\left(a^{\dagger}\right)^{*}\right) \rrbracket=\llbracket\left(a^{\dagger}, a^{*} \cap\left(a^{\dagger} \Rightarrow a\right)\right) \rrbracket$. Therefore, $i\left(a^{\dagger}\right)=(i(a))^{\dagger}$.

The following results are immediate consequence of Theorems 4.4, 4.8 and 4.9.

COROLlary 4.10

Let $\{\mathbb{A}, \mathbb{B}\} \in M_{1}$ and $\mathbf{B}=\left(B, \wedge, \vee, \sim,{ }^{\prime}, 1\right) \in \mathbb{B}$. Then $\mathbf{B}$ is isomorphic to a subalgebra of $\mathbf{V}_{k}(\mathbf{s H}(\mathbf{B}))$.

COROLLARY 4.11

Let $\{\mathbb{A}, \mathbb{B}\} \in M_{1}$ and $\mathbf{A}=\left(A, \cap, \cup, \Rightarrow,,^{\dagger}, \perp, \top\right) \in \mathbb{A}$. Then $\mathbf{A}$ is isomorphic to $\mathbf{s H}\left(\mathbf{V}_{k}(\mathbf{A})\right)$.

The following lemma give some properties involving the subvarieties of dually hemimorphic semiNelson algebras presented in this paper.

LEMMA 4.12

(1) $\mathbb{D M N} \subset \mathbb{D M S N} \subset \mathbb{D} m s \mathbb{S N} \subset \mathbb{O C K} \mathbb{S N} \subset \mathbb{D H M S N}$,

(2) $\mathbb{D P C N} \subset \mathbb{D P C S N} \subset \mathbb{D Q D D S N} \subset \mathbb{D S D S N} \subset \mathbb{D H M} \mathbb{M}$,

(3) $\mathbb{D Q D B N N} \subset \mathbb{D Q D D S N}$,

(4) $\mathbb{D S S N} \subset \mathbb{D Q} \mathbb{Q S N}$,

(5) $\mathbb{B D Q} \mathbb{Q S N} \subset \mathbb{D Q S S N}$, 
(6) $\mathbb{D P} \mathbb{C N} \subset \mathbb{D H M N} \subset \mathbb{D H} \mathbb{M} \mathbb{N}$,

(7) $\mathbb{D M N} \subset \mathbb{D H M N}$,

(8) $\quad \mathbb{D S C S N} \subset \mathbb{D H M} \mathbb{M N}$.

ProOF. The inclusions are an immediate consequence of Lemma 2.4 and Corollaries 4.10 and 4.11.

The following algebra is a dually hemimorphic semi-Nelson algebras, where the operations are defined as follow:



In particular, taking into account the previous algebra it is possible to show that $\mathbb{O C K \mathbb { K }} \neq$ $\mathbb{D H M S H}, \mathbb{D Q S S H} \neq \mathbb{D S S H}$ and $\mathbb{D Q S S H} \neq \mathbb{B D Q S S H}$. Then it follows from Corollaries 4.10 and 4.11 that $\mathbb{O C K S N} \neq \mathbb{D H M} \mathbb{S N}, \mathbb{D Q S S N} \neq \mathbb{D S S N}$ and $\mathbb{D Q S S N} \neq \mathbb{B D Q S S N}$. The rest of the inclusions are strict, and this fact can be proved taking into account the examples given in Section 3.

\subsection{Dually hemimorphic centered semi-Nelson algebras}

We write $\mathbb{S N}_{c}$ for the category whose objects are algebras $(T, \wedge, \vee, \rightarrow, \sim, c, 0,1)$ of type $(2,2,2,1,0,0,0)$, where $(T, \wedge, \vee, \rightarrow, \sim, 1)$ is a semi-Nelson algebra, $0=\sim 1$ and $\mathrm{c}$ satisfies that $c=\sim c$. The morphisms of $\mathbb{S N}_{c}$ are the algebra homomorphisms. In [7] it was proved that there exists a categorical equivalence between $\mathbb{S N}_{c}$ and $\mathbb{S H}$. More precisely, if $\mathbf{A} \in \mathbb{S N c}$ then $\mathbf{s H}(\mathbf{A})=(A / \equiv, \cap, \cup, \Rightarrow, \perp, \top) \in \mathbb{S H}$ and if $f: \mathbf{A} \rightarrow \mathbf{B} \in \mathbb{S N}_{c}$ then $\mathbf{s H}(f): \mathbf{s H}(\mathbf{A}) \rightarrow \mathbf{s H}(\mathbf{B})$ given by $(\mathbf{s H}(f))(\llbracket a \rrbracket)=\llbracket f(a) \rrbracket$ is a morphism in $\mathbb{S H}$. If $\mathbf{A} \in \mathbb{S H}$ then $\mathbf{V}_{k}(\mathbf{A})=(\mathrm{K}(A), \wedge, \vee, \rightarrow$ $, \sim, \mathrm{c}, 0,1) \in \mathbb{S N}_{c}$, where $\mathrm{c}$ is defined as $\mathrm{c}:=(0,0)$. If $f: \mathbf{A} \rightarrow \mathbf{B}$ is a morphism in $\mathbb{S H}$ then $\mathbf{V}_{k}(f): \mathbf{V}_{k}(\mathbf{A}) \rightarrow \mathbf{V}_{k}(\mathbf{B})$ given by $\left(\mathbf{V}_{k}(f)\right)(a, b)=(f(a), f(b))$ is a morphism in $\mathbb{S N}_{c}$. For $A \in \mathbb{S N}_{c}$ we have that the map $h: \mathbf{A} \rightarrow\left(\mathbf{V}_{k}(\mathbf{s H}(A))\right)$ defined by $h(a)=(\llbracket a \rrbracket, \llbracket \sim a \rrbracket)$ is an isomorphism. For $A \in \mathbb{S H}$ we have that the map $i: \mathbf{A} \rightarrow \mathbf{s H}\left(\mathbf{V}_{k}(\mathbf{A})\right)$ given by $i(a)=\llbracket\left(a, a^{*}\right) \rrbracket$ is an isomorphism.

\section{REMARK 4.13}

The functors sH $: \mathbb{S N}_{c} \rightarrow \mathbb{S H}$ and $\mathbf{V}_{k}: \mathbb{S H} \rightarrow \mathbb{S N}_{c}{ }^{1}$ establish a categorical equivalence between $\mathbb{S N}_{c}$ and $\mathbb{S H I}$ with natural isomorphisms $h$ and $i$.

We write $\mathbb{D H} \mathbb{M S N} c$ in order to define the category whose objects are algebras $(T, \wedge, \vee, \rightarrow, \sim$ $\left.,^{\prime}, \mathrm{c}, 0,1\right)$ of type $(2,2,2,1,1,0,0,0)$ such that $(T, \wedge, \vee, \rightarrow, \sim, \mathrm{c}, 0,1) \in \mathbb{S N}_{c}$ and $(T, \wedge, \vee, \rightarrow, \sim$ $,, 1) \in \mathbb{D H} \mathbb{H} \mathbb{S N}$.

Let $f: \mathbf{A} \rightarrow \mathbf{B}$ be a morphism in $\mathbb{D H M S N}$. It is immediate that $\mathbf{s H}(f): \mathbf{s H}(\mathbf{A}) \rightarrow \mathbf{s H}(\mathbf{B})$ is a morphism in $\mathbb{D H} \mathbb{M} \mathbb{S H}$. Conversely, if $f: \mathbf{A} \rightarrow \mathbf{B}$ is a morphism in $\mathbb{D H M} \mathbb{M} H$ then straightforward computations show that $\mathbf{V}_{k}(f): \mathbf{V}_{k}(\mathbf{A}) \rightarrow \mathbf{V}_{k}(\mathbf{B})$ is a morphism in $\mathbb{D H M S N}$. Thus, we have functors $\mathbf{s H}: \mathbb{D H} \mathbb{H} \mathbb{S N} c \rightarrow \mathbb{S H}$ and $\mathbf{V}_{k}: \mathbb{S H} \rightarrow \mathbb{D H M S N}$.

\footnotetext{
${ }^{1}$ Note that we are also abusing notation here with the name of the previous two functors. We believe it is clear which is the corresponding functor considered in each case.
} 
THEOREM 4.14

The functors $\mathbf{s H}: \mathbb{D H M S N} c \rightarrow \mathbb{D H} \mathbb{M S H}$ and $\mathbf{V}_{k}: \mathbb{D H M S H} \rightarrow \mathbb{D H M S N} c$ establish a categorical equivalence between $\mathbb{D H M S N} c$ and $\mathbb{D H M} \mathbb{M} \mathbb{H}$ with natural isomorphisms $h$ and $i$.

PROOF. It follows from Remark 4.13, Theorem 4.8 and Theorem 4.9.

\section{Deductive systems and congruences in $\mathbb{D H M S N}$}

In [10] it was proved that given a semi-Nelson algebra, the set of its congruences is in bijection with a subclass of its deductive systems. In this section we obtain a similar property for the case of dually hemimorphic semi-Nelson algebras.

\section{DEFINITION 5.1}

Let $\mathbf{A}=\left(A, \wedge, \vee, \rightarrow, \sim,{ }^{\prime}, 1\right) \in \mathbb{D H} \mathbb{M} \mathbb{S N}$. A subset $D \subseteq A$ is an $N$-deductive system of $A$ if for every $a, b \in A$, the following conditions are satisfied:

Ds1) $1 \in D$,

$D s 2)$ if $a, a \rightarrow_{N} b \in D$ then $b \in D$.

We say that $D$ is an $N^{\prime}$-deductive system if satisfies the following additional condition:

$D s 3)$ if $a \in D$ then $\left(a^{\prime}\right) \rightarrow_{N}(\sim 1) \in D$.

Let $(A, \wedge, \vee, 1)$ be a lattice with last element and $F \subseteq L$. Recall that $F$ is said to be a filter of $L$ if the following conditions are satisfied: (i) $1 \in F$; (ii) if $x \leq y$ and $x \in F$ then $y \in F$; and (iii) if $x, y \in F$ then $x \wedge y \in F$. The following three lemmas involve properties of deductive systems. See [10, Lemma 6.3], [10, Lemma 6.5] and [10, Lemma 6.6], respectively.

LEMMA 5.2

Let $\mathbf{A}=\left(A, \wedge, \vee, \rightarrow, \sim,{ }^{\prime}, 1\right) \in \mathbb{D H} \mathbb{M} \mathbb{S N}$. If $D$ is an $N$-deductive system of $A$ then $D$ is a filter.

LEMMA 5.3

Let $\mathbf{A}=\left(A, \wedge, \vee, \rightarrow, \sim,,^{\prime}, 1\right) \in \mathbb{D H M S N}$ and $D$ an $N$-deductive system. If $a \rightarrow_{N} b \in D$ and $b \rightarrow_{N} c \in D$, then $a \rightarrow_{N} c \in D$.

LEMMA 5.4

Let $\mathbf{A}=\left(A, \wedge, \vee, \rightarrow, \sim,{ }^{\prime}, 1\right) \in \mathbb{D H M} \mathbb{S N}$ and $D$ an $N$-deductive system of $A$. If $a, b \in A$, then

$$
a \rightarrow b \in D \text { and } b \rightarrow a \in D \text { if and only if } a \rightarrow_{N} b \in D \text { and } b \rightarrow_{N} a \in D .
$$

In what follows we will see that every $N^{\prime}$-deductive system of a dually hemimorphic semi-Nelson algebra determines a congruence. Let $\mathbf{A}=\left(A, \wedge, \vee, \sim,{ }^{\prime}, 1\right) \in \mathbb{D} \mathbb{H M S N}$ and $D$ an $N^{\prime}$-deductive system of $A$. In $A$ we can define the following binary relation:

$$
a \equiv_{D} b \text { if and only if } a \rightarrow b, b \rightarrow a, \sim a \rightarrow \sim b, \sim b \rightarrow \sim a \in D .
$$

Note that it follows from Lemma 5.4 that the definition of $\equiv_{D}$ is equivalent to the following one:

$$
a \equiv_{D} b \text { if and only if } a \rightarrow_{N} b, b \rightarrow_{N} a, \sim a \rightarrow_{N} \sim b, \sim b \rightarrow_{N} \sim a \in D .
$$

The following lemma will be used to prove that $\equiv_{D}$ preserves the unary operation '. 
LEMMA 5.5

Let $\mathbf{A}=\left(A, \wedge, \vee, \rightarrow, \sim,{ }^{\prime}, 1\right) \in \mathbb{D} \mathbb{H M S N}, D$ an $N^{\prime}$-deductive system of $A$ and $a, b \in A$ such that $a \equiv_{D} b$. Then the following conditions are satisfied:

(1) $a^{\prime} \rightarrow_{N} b^{\prime} \in D$

(2) $\left(a^{\prime} \rightarrow a\right) \rightarrow\left(b^{\prime} \rightarrow b\right) \in D$,

(3) $\left(\sim a^{\prime}\right) \rightarrow_{N}\left(\sim b^{\prime}\right) \in D$.

PROOF.

(1) By hypothesis we have that $a \rightarrow_{N} b, b \rightarrow_{N} a, \sim a \rightarrow_{N} \sim b, \sim b \rightarrow_{N} \sim a \in D$. It follows from (5) of Lemma 3.3 that $a^{\prime} \rightarrow_{N}\left((a \rightarrow b)^{\prime} \vee(b \rightarrow a)^{\prime} \vee a^{\prime}\right)=1$. Besides, it follows from (5) of Lemma 3.3 that $\left((a \rightarrow b)^{\prime} \vee(b \rightarrow a)^{\prime} \vee a^{\prime}\right) \rightarrow_{N}((a \rightarrow b) \wedge(b \rightarrow a) \wedge a)^{\prime}=1$. Taking into account (8) of Lemma 3.3 we deduce the equality

$$
a^{\prime} \rightarrow_{N}((a \rightarrow b) \wedge(b \rightarrow a) \wedge a)^{\prime}=1 .
$$

By equation (E31) we obtain that $((a \rightarrow b) \wedge(b \rightarrow a) \wedge a)^{\prime} \rightarrow((a \rightarrow b) \wedge(b \rightarrow a) \wedge b)^{\prime}=1$, so by (2) and (12) of Lemma 3.3 we have that

$$
((a \rightarrow b) \wedge(b \rightarrow a) \wedge a)^{\prime} \rightarrow_{N}((a \rightarrow b) \wedge(b \rightarrow a) \wedge b)^{\prime}=1 .
$$

The item (16) of Lemma 3.3 implies that

$$
((a \rightarrow b) \wedge(b \rightarrow a) \wedge b)^{\prime} \rightarrow_{N}\left((a \rightarrow b)^{\prime} \vee(b \rightarrow a)^{\prime} \vee b^{\prime}\right)=1 .
$$

Thus, it follows from (8) of Lemma 3.3 applied in (5.1), (5.2) and (5.3) that $a^{\prime} \rightarrow_{N}((a \rightarrow$ $\left.b)^{\prime} \vee(b \rightarrow a)^{\prime} \vee b^{\prime}\right)=1$. Hence,

$$
a^{\prime} \rightarrow_{N}\left((a \rightarrow b)^{\prime} \vee(b \rightarrow a)^{\prime} \vee b^{\prime}\right) \in D .
$$

Since $a \rightarrow b \in D$ then $(a \rightarrow b)^{\prime} \rightarrow_{N}(\sim 1) \in D$. By (9) of Lemma 3.3, $(\sim 1) \rightarrow_{N} b^{\prime}=1 \in$ $D$. Then Lemma 5.3 implies that $(a \rightarrow b)^{\prime} \rightarrow_{N} b^{\prime} \in D$. Analogously, $(b \rightarrow a)^{\prime} \rightarrow_{N} b^{\prime} \in D$. Besides, $b^{\prime} \rightarrow_{N} b^{\prime}=1 \in D$. In consequence, taking into account (6) of Lemma 3.3 we obtain that

$\left((a \rightarrow b)^{\prime} \vee(b \rightarrow a)^{\prime} \vee b^{\prime}\right) \rightarrow_{N} b^{\prime}=\left((a \rightarrow b)^{\prime} \rightarrow_{N} b^{\prime}\right) \wedge\left((b \rightarrow a)^{\prime} \rightarrow_{N} b^{\prime}\right) \wedge\left(b^{\prime} \rightarrow_{N} b^{\prime}\right) \in D$

because $D$ is a filter by Lemma 5.2. By using Lemma 5.3 applied in (5.4) and (5.5) we conclude that $a^{\prime} \rightarrow_{N} b^{\prime} \in D$.

(2) It follows from item (1) of this lemma that $a^{\prime} \rightarrow b^{\prime} \in D$ and $b^{\prime} \rightarrow a^{\prime} \in D$. By equation (E25) we have that $\left(a^{\prime} \rightarrow_{N} b^{\prime}\right) \rightarrow_{N}\left[\left(b^{\prime} \rightarrow_{N} a^{\prime}\right) \rightarrow_{N}\left[\left(a^{\prime} \rightarrow a\right) \rightarrow_{N}\left(b^{\prime} \rightarrow a\right)\right]\right]=1 \in D$. Hence,

$$
\left(a^{\prime} \rightarrow a\right) \rightarrow_{N}\left(b^{\prime} \rightarrow a\right) \in D .
$$

It follows from (E26) that $\left(a^{\prime} \rightarrow_{N} b^{\prime}\right) \rightarrow_{N}\left[\left(b^{\prime} \rightarrow_{N} a^{\prime}\right) \rightarrow_{N}\left[\left(b^{\prime} \rightarrow a\right) \rightarrow_{N}\left(b^{\prime} \rightarrow b\right)\right]\right]=$ $1 \in D$ and

$$
\left(b^{\prime} \rightarrow a\right) \rightarrow_{N}\left(b^{\prime} \rightarrow b\right) \in D .
$$

Then, by Lemma 5.3 applied in (5.6) and (5.7) we obtain that $\left(a^{\prime} \rightarrow a\right) \rightarrow\left(b^{\prime} \rightarrow b\right) \in D$.

(3) It follows from (2) that $\left(a^{\prime} \rightarrow a\right) \rightarrow\left(b^{\prime} \rightarrow b\right) \in D$. Since $a \equiv_{D} b$ then $(\sim a) \rightarrow_{N}(\sim b) \in D$. By $(5)$ of Lemma 3.3, $\left((\sim a) \wedge\left(a^{\prime} \rightarrow a\right)\right) \rightarrow_{N}\left(a^{\prime} \rightarrow a\right)=1 \in D$. It follows from Lemma 5.3 that $\left((\sim a) \wedge\left(a^{\prime} \rightarrow a\right)\right) \rightarrow_{N}\left(b^{\prime} \rightarrow b\right) \in D$. Similarly, $\left((\sim a) \wedge\left(a^{\prime} \rightarrow a\right)\right) \rightarrow_{N}(\sim b) \in D$. Straightforward computations based on (4) of Lemma 3.3 show that $\left((\sim a) \wedge\left(a^{\prime} \rightarrow a\right)\right) \rightarrow_{N}$ 
$\left((\sim b) \wedge\left(b^{\prime} \rightarrow b\right)\right)=\left(\left((\sim a) \wedge\left(a^{\prime} \rightarrow a\right)\right) \rightarrow_{N}(\sim b)\right) \wedge\left(\left((\sim a) \wedge\left(a^{\prime} \rightarrow a\right)\right) \rightarrow_{N}\left(b^{\prime} \rightarrow\right.\right.$ $b)) \in D$ because $D$ is a filter, i.e.

$$
\left((\sim a) \wedge\left(a^{\prime} \rightarrow a\right)\right) \rightarrow_{N}\left((\sim b) \wedge\left(b^{\prime} \rightarrow b\right)\right) \in D .
$$

The assertion $\left(\sim a^{\prime}\right) \rightarrow\left(\sim a \wedge\left(a^{\prime} \rightarrow a\right)\right)=1$ holds in the algebra by (E32). Then by (2) and (12) of Lemma 3.3 we deduce that

$$
\left(\sim a^{\prime}\right) \rightarrow_{N}\left(\sim a \wedge\left(a^{\prime} \rightarrow a\right)\right)=1 \in D .
$$

Taking into account Lemma 5.3 applied in (5.8) and (5.9) we obtain that

$$
\left(\sim a^{\prime}\right) \rightarrow_{N}\left((\sim b) \wedge\left(b^{\prime} \rightarrow b\right)\right) \in D .
$$

Using (E33) we have that $\left((\sim b) \wedge\left(b^{\prime} \rightarrow b\right)\right) \rightarrow\left(\sim b^{\prime}\right)=1$. Thus, by (2) and (12) of Lemma 3.3,

$$
\left((\sim b) \wedge\left(b^{\prime} \rightarrow b\right)\right) \rightarrow_{N}\left(\sim b^{\prime}\right)=1 \in D .
$$

Therefore, it follows from (5.3) applied in (5.10) and (5.11) that

$$
\left(\sim a^{\prime}\right) \rightarrow_{N}\left(\sim b^{\prime}\right) \in D .
$$

\section{LEMMA 5.6}

Let $\mathbf{A}=\left(A, \wedge, \vee, \rightarrow, \sim,{ }^{\prime}, 1\right) \in \mathbb{D H M} \mathbb{S}$ and $D$ an $N^{\prime}$-deductive system of $A$. The relation $\equiv_{D}$ is a congruence.

ProOF. It follows from [10, Lemma 6.7] that $\equiv_{D}$ is an equivalence relation compatible with the operations $\sim, \wedge, \vee, \rightarrow$. We will prove that $\equiv_{D}$ also preserves the operation ' . Let $a, b \in A$ such that $a \equiv_{D} b$. It follows from (1) of Lemma 5.5 that $a^{\prime} \rightarrow_{N} b^{\prime} \in D$. Similarly, we have that $b^{\prime} \rightarrow a^{\prime} \in D$. Taking into account (3) of Lemma 5.5 we have that $\left(\sim a^{\prime}\right) \rightarrow_{N}\left(\sim b^{\prime}\right) \in D$. In an analogous way, $\left(\sim b^{\prime}\right) \rightarrow_{N}\left(\sim a^{\prime}\right) \in D$. Therefore, $a^{\prime} \equiv_{D} b^{\prime}$.

Let us now check that every congruence on a dually hemimorphic semi Nelson algebra determines a deductive system. From now on we denote by $\operatorname{Con}(\mathbf{A})$ the congruence lattice of an algebra $\mathbf{A}$. If $\Theta \in \mathbf{C o n}(\mathbf{A})$ we denote by $\llbracket a \rrbracket_{\Theta}$ the class of an element $a \in A$ modulo $\Theta$.

\section{LEMMA 5.7}

Let $\mathbf{A}=\left(A, \wedge, \vee, \rightarrow, \sim,{ }^{\prime}, 1\right) \in \mathbb{D H} \mathbb{M S N}$. If $\Theta \in \mathbf{C o n}(\mathbf{A})$, then $\llbracket 1 \rrbracket \Theta$ is an $N^{\prime}$-deductive system.


Let $a \in A$ such that $(a, 1) \in \Theta$. Since $\Theta$ is a congruence, $\left(a^{\prime} \rightarrow(\sim 1), 1^{\prime} \rightarrow(\sim 1)\right) \in \Theta$. Then by (E30) we obtain that $1^{\prime} \rightarrow(\sim 1)=1$. Therefore, $a^{\prime} \rightarrow(\sim 1) \in \llbracket 1 \rrbracket_{\Theta}$.

The following three results are consequence from Lemma 5.6, Lemma 5.7, [10, Lemma 6.8], [10, Lemma 6.10] and [10, Theorem 6.11].

\section{LEMMA 5.8}

Let $\mathbf{A}=\left(A, \wedge, \vee, \rightarrow, \sim,{ }^{\prime}, 1\right) \in \mathbb{D H} \mathbb{M} \mathbb{S N}$ and $D$ be an $N^{\prime}$-deductive system of $A$. Then $\llbracket 1 \rrbracket_{\equiv_{D}}=D$.

\section{LEMMA 5.9}

Let $\mathbf{A}=\left(A, \wedge, \vee, \rightarrow,{ }^{\prime}, 1\right) \in \mathbb{D H} \mathbb{H} \mathbb{S N}$ and $\Theta \in \mathbf{C o n}(\mathbf{A})$. Then

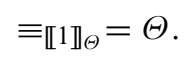

If $\mathbf{A} \in \mathbb{D H M} \mathbb{M}$ we will write $\operatorname{Ded}_{\mathbf{N}}(\mathbf{A})$ for the lattice of $N^{\prime}$-deductive systems of $\mathbf{A}$. 
THEOREM 5.10

Let $\mathbf{A}=\left(A, \wedge, \vee, \rightarrow, \sim,{ }^{\prime}, 1\right) \in \mathbb{D H M S N}$. Then the lattices $\operatorname{Ded}_{\mathbf{N}}(\mathbf{A})$ and $\operatorname{Con}(\mathbf{A})$ are isomorphic.

It follows from [10, Theorem 6.13] that $\mathbb{D H} \mathbb{H} \mathbb{M N}$ is congruence permutable and it follows from $[10$, Corollary 6.14] that $\mathbb{D H M S N}$ is arithmetical.

\section{Final remarks}

In this final section we will prove that the categories $\mathbb{D H M S H}$ and $\mathbb{D H} \mathbb{M} \mathbb{S N}_{c}$ are equivalent by considering an alternative construction to that given in Section 4.

We start with some preliminary definitions and results.

Let $\mathbf{A}=(A, \wedge, \vee, \rightarrow, \sim, \mathrm{c}, 0,1) \in \mathbb{S N}_{c}$ and define the set $\mathrm{C}(A)=\{x \in A: x \geq \mathrm{c}\}$. We have that $\mathbf{C}(\mathbf{A})=(\mathrm{C}(A), \cap, \cup, \Rightarrow, \perp, \top) \in \mathbb{S H}$. For a morphism $f: \mathbf{A} \rightarrow \mathbf{B} \in$ $\mathbb{S N}_{c}$, the map $\mathbf{C}(f): \mathbf{C}(\mathbf{A}) \rightarrow \mathbf{C}(\mathbf{B})$, given by $\mathbf{C}(f)(a)=g(a)$, is a morphism in $\mathbb{S H}$. Moreover, $\mathbf{C}$ is a functor from $\mathbb{S N}_{c}$ to $\mathbb{S H}$. If $\mathbf{A} \in \mathbb{S H}$ then the map $\alpha_{\mathbf{A}}: \mathbf{A} \rightarrow \mathbf{C}\left(\mathbf{V}_{k}(\mathbf{A})\right)$ given by $\alpha_{\mathbf{A}}(a)=(a, 0)$ is an isomorphism in $\mathbb{S H}$. If $\mathbf{A} \in \mathbb{S N}_{c}$, the map $\beta_{\mathbf{A}}: \mathbf{A} \rightarrow$ $\mathbf{V}_{k}(\mathbf{C}(\mathbf{A}))$ given by $\beta_{\mathbf{A}}(a)=(a \vee \mathrm{c}, \sim a \vee \mathrm{c})$ is an isomorphism in $\mathbb{S N}_{c}$. The following is [7, Theorem 3.9].

THEOREM 6.1

The functors $\mathbf{V}_{k}$ and $\mathbf{C}$ establish a categorical equivalence between $\mathbb{S H I}$ and $\mathbb{S N}_{c}$ with natural isomorphisms $\alpha$ and $\beta$.

In what follows we will give some technical properties of $\mathbb{D H M} \mathbb{S N}_{c}$.

LEMMA 6.2

If $\mathbf{A}=\left(A, \cap, \cup, \Rightarrow,{ }^{\dagger}, \perp, \top\right) \in \mathbb{D H M} \mathbb{M H}$, then $\mathbf{V}_{k}(\mathbf{A})$ satisfies the following equalities:

(1) $x^{\prime} \vee \mathrm{c}=(x \vee \mathrm{c})^{\prime}$,

(2) $((x \vee c) \wedge(y \vee c))^{\prime}=(x \vee c)^{\prime} \vee(y \vee c)^{\prime}$,

(3) $\sim x^{\prime} \vee \mathrm{c}=(\sim x \vee \mathrm{c}) \wedge\left((x \vee \mathrm{c})^{\prime} \rightarrow(x \vee \mathrm{c})\right)$.

PROOF.

1. $((a, b) \vee(\perp, \perp))^{\prime}=(a, \perp)^{\prime}=\left(a^{\dagger}, \perp\right)=\left(a^{\dagger}, b \cap\left(a^{\dagger} \Rightarrow a\right)\right) \vee(\perp, \perp)=(a, b)^{\prime} \vee(\perp, \perp)$.

2. $(((a, b) \vee(\perp, \perp)) \wedge((c, d) \vee(\perp, \perp)))^{\prime}=((a, \perp) \wedge(c, \perp))^{\prime}=(a \cap c, \perp)^{\prime}=\left((a \cap c)^{\dagger}, \perp\right) \stackrel{(E 6)}{=}$ $\left(a^{\dagger} \cup c^{\dagger}, \perp\right)=\left(a^{\dagger}, \perp\right) \vee\left(c^{\dagger}, \perp\right)=(a, \perp)^{\prime} \vee(c, \perp)^{\prime}=((a, b) \vee(\perp, \perp))^{\prime} \vee((c, d) \vee(\perp, \perp))^{\prime}$.

3. $(\sim(a, b) \vee(\perp, \perp)) \wedge\left(((a, b) \vee(\perp, \perp))^{\prime} \rightarrow((a, b) \vee(\perp, \perp))\right)=((b, a) \vee(\perp, \perp)) \wedge(((a, b) \vee$ $\left.(\perp, \perp))^{\prime} \rightarrow((a, b) \vee(\perp, \perp))\right)=(b, \perp) \wedge\left((a, \perp)^{\prime} \rightarrow(a, \perp)\right)=(b, \perp) \wedge\left(\left(a^{\dagger}, \perp\right) \rightarrow(a, \perp)\right)=$ $(b, \perp) \wedge\left(a^{\dagger} \Rightarrow a, \perp\right)=\left(b \cap\left(a^{\dagger} \Rightarrow a\right), \perp\right)=\left(b \cap\left(a^{\dagger} \Rightarrow a\right), a^{\dagger}\right) \vee(\perp, \perp)=\sim\left(a^{\dagger}, b \cap\left(a^{\dagger} \Rightarrow\right.\right.$ a)) $\vee(\perp, \perp)=\sim(a, b)^{\prime} \vee(\perp, \perp)$.

The following corollary follows from Lemma 6.2, Theorem 4.8 and the fact that morphisms in $\mathbb{S N}$ between objects in $\mathbb{S N}_{c}$ necessarily preserves c.

COROLLARY 6.3

Let $\mathbf{A}=\left(A, \wedge, \vee, \rightarrow, \sim,{ }^{\prime}, c, 0,1\right) \in \mathbb{D H M} \mathbb{M N} c$. Then the following equalities are satisfied:

(1) $x^{\prime} \vee \mathrm{c}=(x \vee \mathrm{c})^{\prime}$,

(2) $((x \vee c) \wedge(y \vee c))^{\prime}=(x \vee c)^{\prime} \vee(y \vee c)^{\prime}$,

(3) $\sim x^{\prime} \vee \mathrm{c}=(\sim x \vee \mathrm{c}) \wedge\left((x \vee \mathrm{c})^{\prime} \rightarrow(x \vee \mathrm{c})\right)$. 


\section{LEMMA 6.4}

Let $\mathbf{A}=\left(A, \wedge, \vee, \rightarrow, \sim,{ }^{\prime}, \mathrm{c}, 1\right)$ be an algebra of type $(2,2,2,1,1,0,0)$ such that satisfies the following conditions:

(1) $(A, \wedge, \vee, 0,1)$ is a lattice with last element,

(2) $\mathrm{c}=1^{\prime}$

(3) $((x \vee c) \wedge(y \vee c))^{\prime}=(x \vee c)^{\prime} \vee(y \vee c)^{\prime}$.

Then A satisfies $(x \vee c)^{\prime} \geq c$.

PROOF. Let $x \in A$. Then $\mathrm{c} \leq \mathrm{c} \vee(x \vee \mathrm{c})^{\prime} \stackrel{(2)}{=} 1^{\prime} \vee(x \vee \mathrm{c})^{\prime}=(1 \vee \mathrm{c})^{\prime} \vee(x \vee \mathrm{c})^{\prime} \stackrel{(3)}{=}((1 \vee \mathrm{c}) \wedge(x \vee \mathrm{c}))^{\prime}=$ $(x \vee \mathrm{c})^{\prime}$.

\section{LEMMA 6.5}

Let $\mathbf{A}=\left(A, \wedge, \vee, \rightarrow, \sim,{ }^{\prime}, \mathrm{c}, 1\right) \in \mathbb{D} \mathbb{H M S N} c$. Then $\mathbf{A}$ satisfies the following equalities:

(1) $\mathrm{c}^{\prime}=1$,

(2) $\mathrm{c}=1^{\prime}$,

(3) $(x \vee c)^{\prime} \geq \mathrm{c}$.

PROOF.

1. Note that $1 \stackrel{(E 29)}{=}(\sim 1)^{\prime}=(\sim(1 \wedge \mathrm{c}))^{\prime}=((\sim 1) \vee(\sim \mathrm{c}))^{\prime}=((\sim 1) \vee \mathrm{c})^{\prime} \stackrel{3.3(1)}{=} c^{\prime}$.

2. It follows from (E30) that $1^{\prime} \rightarrow(\sim 1)=1$. Then it follow from (2) and (12) of Lemma 3.3 that

$$
1^{\prime} \rightarrow_{N}(\sim 1)=1
$$

By (9) of Lemma 3.3,

$$
(\sim 1) \rightarrow_{N} c=1 .
$$

By (8) of Lemma 3.3 in (6.1) and (6.2) we obtain that

$$
1^{\prime} \rightarrow{ }_{N} c=1 .
$$

By (10) of Lemma 3.3 we have that $(\mathrm{c} \wedge \sim \mathrm{c}) \rightarrow_{N}\left(\sim 1^{\prime}\right)=1$. As $\mathrm{c}=\sim \mathrm{c}$,

$$
(\sim \mathrm{c}) \rightarrow_{N}\left(\sim 1^{\prime}\right)=1
$$

By (7) of Lemma 3.3 applied in (6.3) and (6.4) we obtain that

$$
1^{\prime} \leq \mathrm{c} .
$$

By other hand, $1^{\prime} \vee \mathrm{c} \stackrel{6.3(1)}{=}(1 \vee \mathrm{c})^{\prime}=1^{\prime}$. Thus,

$$
\mathrm{c} \leq 1^{\prime} \text {. }
$$

3. In the previous item we show that $\mathbf{A}$ satisfies the equality $1^{\prime}=\mathrm{c}$. By (2) of Corollary 6.3 we have that $\mathbf{A}$ satisfies the equality $((x \vee c) \wedge(y \vee c))^{\prime}=(x \vee c)^{\prime} \vee(y \vee c)^{\prime}$. So, by Lemma 6.4 we have that

$$
(x \vee \mathrm{c})^{\prime} \geq \mathrm{c} .
$$

\section{LEMMA 6.6}

If $\mathbf{A}=\left(A, \wedge, \vee, \rightarrow, \sim,^{\prime}, c, 1\right) \in \mathbb{D H} \mathbb{M} \mathbb{S} \mathbb{N}_{c}$ then $\mathbf{C}(A)=\left(C(A), \wedge, \vee, \rightarrow,^{\prime}, c, 1\right) \in \mathbb{D H M} \mathbb{M} H$. 
ProOF. By Lemma 6.5 the equality $(x \vee \mathrm{c})^{\prime} \geq \mathrm{c}$ is satisfied. Hence, the operation ' is well defined in $\mathbf{C}(A)$. It follows from (1) and (2) of Lemma 6.5 that $\mathbf{C}(A)$ verifies (E4) and (E5). Besides, by (2) of Lemma 6.3 we have that $\mathbf{C}(A)$ satisfies (E6).

It is immediate that $\mathbf{C}$ is a functor from $\mathbb{D H M S N}_{c}$ and $\mathbb{D H M S H}$.

LEMMA 6.7

(a) Let $\mathbf{A} \in \mathbb{D} H \mathbb{M} \mathbb{S H}$ and $a \in A$. Then $\alpha_{\mathbf{A}}\left(a^{\dagger}\right)=\alpha_{\mathbf{A}}(a)^{\prime}$.

(b) Let $\mathbf{A} \in \mathbb{D} \mathbb{H M S N} c$ and $a \in \mathbf{A}$. Then $\beta_{\mathbf{A}}\left(a^{\prime}\right)=\beta_{\mathbf{A}}(a)^{\prime}$.

PROOF.

(a) $\quad \alpha_{\mathbf{A}}\left(a^{\dagger}\right)=\left(a^{\dagger}, 0\right)=(a, 0)^{\prime}=\alpha_{\mathbf{A}}(a)^{\prime}$.

(b) $\beta_{\mathbf{A}}\left(a^{\prime}\right)=\left(a^{\prime} \vee \mathrm{c}, \sim a^{\prime} \vee \mathrm{c}\right) \stackrel{6.3(1)}{=}\left((a \vee c)^{\prime}, \sim a^{\prime} \vee \mathrm{c}\right) \stackrel{6.3(3)}{=}\left((a \vee \mathrm{c})^{\prime},(\sim a \vee \mathrm{c}) \wedge\left((a \vee \mathrm{c})^{\prime} \rightarrow\right.\right.$ $(a \vee \mathrm{c})))=(a \vee \mathrm{c}, \sim a \vee \mathrm{c})^{\prime}=\beta_{\mathrm{A}}(a)^{\prime}$.

Therefore, it follows from Lemma 6.7 and Theorem 6.1 the following result.

THEOREM 6.8

The functors $\mathbf{V}_{k}$ and $\mathbf{C}$ establish a categorical equivalence between $\mathbb{D H M S H}$ and $\mathbb{D H} \mathbb{M} \mathbb{S N}_{c}$ with natural isomorphisms $\alpha$ and $\beta$.

In conclusion, the present paper is a continuation of a work started in [10], where the correspondence between the variety of Heyting algebras and the variety of Nelson algebras was generalized in the framework of the varieties of semi-Heyting algebras and of semi-Nelson algebras, respectively. More precisely, the main goal of this paper was to extend the results of [10] in the framework of the variety of dually hemimorphic semi-Heyting algebras [19].

\section{Acknowledgements}

The authors acknowledge many helpful comments from the anonymous referee, which improved the presentation of this paper. This work was partially supported by Consejo Nacional de Investigaciones Científicas y Técnicas.

\section{References}

[1] M. Abad, J. M. Cornejo and J. P. Díaz Varela. The variety generated by semi-Heyting chains. Soft Computing, 15, 721-728, 2010.

[2] M. Abad, J. M. Cornejo and J. P. Díaz Varela. The variety of semi-Heyting algebras satisfying the equation $(0 \rightarrow 1)^{\dagger} \vee(0 \rightarrow 1)^{\dagger \dagger}=1$. Reports on Mathematical Logic, 46, 75-90, 2011.

[3] M. Abad, J. M. Cornejo and J. P. Díaz Varela. Free-decomposability in varieties of semi-Heyting algebras. Mathematical Logic Quarterly, 58, 168-176, 2012.

[4] M. Abad, J. M. Cornejo and J. P. Díaz Varela. Semi-Heyting algebras term-equivalent to Gödel algebras. Order, 30, 625-642, 2013.

[5] D. Castaño and J. M Cornejo. Gentzen-style sequent calculus for semi-intuitionistic logic. Studia Logica, 104, 1245-1265, 2016.

[6] J. M. Cornejo. Semi-intuitionistic logic. Studia Logica, 98, 9-25, 2011.

[7] J. M. Cornejo and H. J. San Martín. A categorical equivalence between semi-Heyting algebras and centered semi-Nelson algebras. Logic Journal of the IGPL, 26, 408-428, 2018. 
[8] J. M. Cornejo and H. P. Sankappanavar. A Logic for Dually Hemimorphic Semi-Heyting Algebras and Axiomatic Extensions. (arXiv:1908.02403v1 [math.LO]).

[9] J. M. Cornejo and I. D. Viglizzo. On some semi-intuitionistic logics. Studia Logica, 103, 303344, 2015.

[10] J. M. Cornejo and I. Viglizzo. Semi-Nelson algebras. Order, 35, 23-45, 2018.

[11] J. A. Kalman. Lattices with involution. Transactions of the American Mathematical Society, 87, 485-491, 1958.

[12] M Kracht. On extensions of intermediate logics by strong negation. Journal of Philosophical Logic, 27, 49-73, 1998.

[13] G. C. Moisil. Logique modale. Disquisitiones Mathematicae et Physica, 2, 3-98, 1942.

[14] A. Monteiro. Sur les algèbres de Heyting symétriques. Portugaliae Mathematica, 39, 1-237, 1980. Special Issue in honor of António Monteiro.

[15] D. Nelson. Constructible falsity. The Journal of Symbolic Logic, 14, 16-26, 1949.

[16] S. Odintsov. On the representation of N4-lattices. Studia Logica, 76, 385-405, 2004.

[17] H. Rasiowa. $\mathcal{N}$-lattices and constructive logic with strong negation. Fundamenta Mathematicae, 46, 61-80, 1958.

[18] U. Rivieccio. Implicative twist-structures. Algebra Universalis, 71, 155-186, 2014.

[19] H. P. Sankappanavar. Expansions of semi-Heyting algebras I: discriminator varieties. Studia Logica, 98, 27-81, 2011.

[20] H. P. Sankappanavar. Heyting algebras with a dual lattice endomorphism. Zeitschrift fr Mathematische Logik und Grundlagen der Mathematik, 33, 565-573, 1987.

[21] H. P. Sankappanavar. Semi-Heyting algebras: an abstraction from Heyting algebras. Actas del IX Congreso Dr. Antonio A. R. Monteiro, 33-66, 2007.

[22] A. Sendlewski. Nelson algebras through Heyting ones: I. Studia Logica, 49, 105-126, 1990.

[23] M. Sholander. Postulates for distributive lattices. Canadian Journal of Mathematics, 3, 28-30, 1951.

[24] D. Vakarelov. Notes on $\mathcal{N}$-lattices and constructive logic with strong negation. Studia Logica, 36, 109-125, 1977.

[25] I. Viglizzo. Álgebras de Nelson. Magister dissertation in Mathematics, Instituto de Matemática de Bahía Blanca, Universidad Nacional del Sur, 1999. https://sites.google.com/site/viglizzo/ viglizzo99nelson.

Received 17 July 2019 